\title{
Operating a passive on-chip superconducting circulator: Device control and quasiparticle effects
}

\author{
Dat Thanh Le $\odot,{ }^{1, *}$ Clemens Müller $\odot,{ }^{1,2, \dagger}$ Rohit Navarathna, ${ }^{1}$ Arkady Fedorov, ${ }^{1}$ and T. M. Stace ${ }^{1, \ddagger}$ \\ ${ }^{1}$ ARC Centre for Engineered Quantum System, School of Mathematics and Physics, University of Queensland, Brisbane, \\ Queensland 4072, Australia \\ ${ }^{2}$ IBM Quantum, IBM Research-Zurich, 8803 Rüschlikon, Switzerland
}

(Received 24 February 2021; revised 2 August 2021; accepted 4 December 2021; published 23 December 2021)

\begin{abstract}
Microwave circulators play an important role in quantum technology based on superconducting circuits. The conventional circulator design, which employs ferrite materials, is bulky and involves strong magnetic fields, rendering it unsuitable for integration on superconducting chips. One promising design for an on-chip superconducting circulator is based on a passive Josephson-junction ring. In this paper, we consider two operational issues for such a device: circuit tuning and the effects of quasiparticle tunneling. We compute the scattering matrix using adiabatic elimination and derive the parameter constraints to achieve optimal circulation. We then numerically optimize the circulator performance over the full set of external control parameters, including gate voltages and flux bias, to demonstrate that this multidimensional optimization converges quickly to find optimal working points. We also consider the possibility of quasiparticle tunneling in the circulator ring and how it affects signal circulation. Our results form the basis for practical operation of a passive on-chip superconducting circulator made from a ring of Josephson junctions.
\end{abstract}

DOI: 10.1103/PhysRevResearch.3.043211

\section{INTRODUCTION}

Microwave circulators are widely used in experiments with superconducting circuits [1]. They break Lorentz reciprocity [2] and facilitate unidirectional signal propagation, thus protecting fragile quantum systems from noise and enabling discrimination between input and output fields for quantumlimited amplification [3]. Commercially available circulators are typically realized using ferrite materials and the Faraday effect to induce nonreciprocity [4]. This approach necessitates device dimensions of the order of the microwave wavelength, which poses a practical difficulty for integrating circulators with chip-based superconducting circuits. Furthermore, the strong magnetic fields in conventional circulators are incompatible with sensitive superconducting devices. Hence, a great deal of effort has been devoted to implementation of ferrite-magnet-free circulators exploiting various physical mechanisms, such as the quantum Hall effect [5,6], interfering parametric processes [7-9], temporal modulation of couplings $[3,10-15]$, noncommutation between frequency conversion and delay [16], and reservoir engineering [17,18].

Recently, Müller et al. [19] analyzed a proposal for a superconducting Josephson-junction-ring circulator whose working principle parallels that of conventional ferrite circulators. The Josephson-junction ring is promising for quantum simulation

\footnotetext{
*thanhdat.le@uq.net.au

${ }^{\dagger}$ Present address: Zurich Instruments AG, Zurich, Switzerland.

${ }^{\ddagger}$ stace@physics.uq.edu.au
}

Published by the American Physical Society under the terms of the Creative Commons Attribution 4.0 International license. Further distribution of this work must maintain attribution to the author(s) and the published article's title, journal citation, and DOI. and potential applications that require nonreciprocity [20-22], as it is compatible with on-chip superconducting circuits and works passively, i.e., does not require an external drive. The physics behind nonreciprocal signal circulation in this device is the Aharonov-Bohm effect [19,20]. This effect (and the signal circulation) is strongly dependent on the external charge and flux biases, the signal frequency, as well as fabrication imperfections of the device parameters.

Because optimal circulator performance requires precise tuning of the external parameters, we here address two related operational issues: (i) tuning to the ideal working point in the multidimensional space of the control parameters, and (ii) the effect of quasiparticle-induced fluctuations $[23,24]$ on the circulator. Tuning the device will likely be necessary in all implementations and, given the numerous independent control parameters, issue (i) may present an operational challenge. Issue (ii) has not been touched upon in Ref. [19], which only showed resilience of signal circulation against perturbations in external biases. Unlike these parameter perturbations, tunneling of a quasiparticle into and/or out of a superconducting island shifts the charge bias on that island by one electron worth of charge [25-27], which detunes the circulator away from its optimal operating points and impairs the tuning procedure (i). Understanding the effect of quasiparticles is a step towards mitigating their impact on the device operation.

Therefore, in this paper we first consider optimization of the superconducting circulator proposed in Ref. [19]; that is, we describe a protocol for tuning the device in the multidimensional parameter space to find optimal operating points. To do this, we employ the adiabatic elimination procedure to extract semianalytic expressions for the scattering matrix elements in the SLH input-output formalism [28,29]. This allows us to deduce quantitative conditions for optimal circulation. We also present numerical optimization results based 
on a full treatment of the multilevel scattering problem. The numerics are found to be in excellent agreement with the semianalytical predictions specifying optimal working points for the circulator.

Second, we address the effect of quasiparticles on the circulator efficiency. We show that due to tunneling of quasiparticles between different pairs of superconducting islands the Josephson-junction-ring circulator in Ref. [19] has four accessible charge-parity sectors. Given the same working conditions and parameters, these sectors circulate signals with different efficiencies. Stochastic jumps among the sectors caused by quasiparticle tunneling events then may result in unstable operation of the circulator device. To mitigate these fluctuations, we propose to employ quasiparticle-trapping techniques $[24,25,30-33]$ to suppress quasiparticle population.

The structure of this paper is as follows. In Sec. II we present the circuit design of the passive on-chip superconducting circulator along with the SLH formalism to numerically calculate the scattering matrix elements. Then in Sec. III we derive the scattering matrix elements exploiting the adiabatic elimination technique and determine the conditions for optimal circulation, followed by numerical optimization in Sec. IV. Section V analyzes quasiparticle tunneling in the circulator system. The paper is concluded in Sec. VII. Appendixes provide detailed calculations and additional information for the results in the main text.

\section{CIRCUIT DESIGN AND SLH FORMALISM}

In this section we present the circuit design of the ring circulator, its working principle, the SLH formalism to compute the scattering matrix, and the notations used throughout the paper. Many details of these can be found in Ref. [19]. The circulator circuit, depicted in Fig. 1(a), is a superconducting ring segmented into three superconducting islands by three Josephson junctions, each of which is described by a Josephson energy $E_{J_{j}}$ and a junction capacitance $C_{J_{j}}(j=1,2,3)$. The three islands are represented by the superconducting phases $\hat{\phi}_{j}$ and their conjugate charges $\hat{n}_{j}$; they are biased by external voltages $V_{x_{j}}$ with gate capacitances $C_{x_{j}}$ and coupled to three external waveguides by coupling capacitances $C_{c_{j}}$. The circulator ring is threaded by an external flux $\Phi_{x}$. Input fields $b_{\text {in, } j}$ propagate along the waveguides, interact with the ring, and scatter into output fields $b_{\text {out }, j}$.

To begin, we consider the case of a symmetric Josephsonjunction ring, that is, $E_{J_{j}}=E_{J}$ and $C_{J_{j}}=C_{J}$, and further assume that $C_{x_{j}}=C_{x}$ and $C_{c_{j}}=C_{c}$. We consider asymmetries later. As derived in Appendix A, the circulator ring Hamiltonian is

$$
\begin{aligned}
\hat{H}_{\text {ring }}= & \frac{(2 e)^{2}}{2}\left(\hat{\mathbf{n}}-\mathbf{n}_{x}\right) \mathbb{C}^{-1}\left(\hat{\mathbf{n}}-\mathbf{n}_{x}\right) \\
& -E_{J} \sum_{j=1}^{3} \cos \left(\hat{\phi}_{j}-\hat{\phi}_{j+1}-\frac{1}{3} \phi_{x}\right),
\end{aligned}
$$

where $\hat{\mathbf{n}}=\left\{\hat{n}_{1}, \hat{n}_{2}, \hat{n}_{3}\right\}, \quad \mathbf{n}_{x}=\left\{n_{x_{1}}, n_{x_{2}}, n_{x_{3}}\right\} \quad$ with $\quad n_{x_{j}}=$ $C_{x_{j}} V_{x_{j}} /(2 e)$ the (dimensionless) charge bias on the island $j, \phi_{x}=2 \pi \Phi_{x} / \Phi_{0}$ is the reduced flux bias which has been shared equally by the three Josephson junctions with $\Phi_{0}=h /(2 e)$ the superconducting quantum flux, and $\mathbb{C}$ is
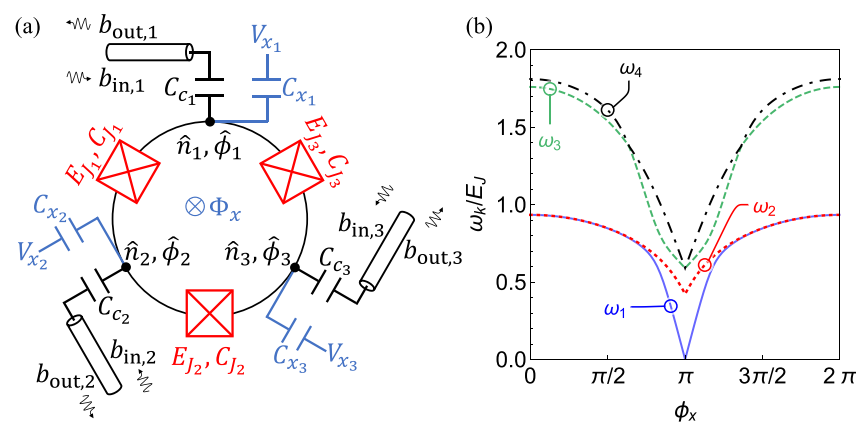

FIG. 1. (a) Schematic circuit design of the passive on-chip superconducting circulator proposed in Ref. [19]. The device comprises three superconducting islands which are represented by the numbers of Cooper pairs $\hat{n}_{j}$ and the superconducting phases $\hat{\phi}_{j}(j=1,2,3)$ on each island. They are connected by three Josephson junctions with Josephson energies $E_{J_{j}}$ and junction capacitances $C_{J_{j}}$. Each island is biased by an external voltage $V_{x_{j}}$ via a gate capacitance $C_{x_{j}}$ and coupled capacitively to a waveguide via a coupling capacitance $C_{c_{j}}$. The whole circulator loop is threaded by a central external flux $\Phi_{x}$ as well. (b) First four excited-state energies $\omega_{k}(k=1,2,3,4)$ of the circulator ring versus the reduced external flux $\phi_{x}$ for a symmetric circuit (i.e., $E_{J_{j}}=E_{J}, C_{J_{j}}=C_{J}, C_{x_{j}}=C_{x}$, and $C_{c_{j}}=C_{c}$ ). The eigenenergies are computed by numerically solving the eigensystem of $\hat{H}_{\text {ring }}$ given in Eq. (4) with $E_{C_{\Sigma}} / E_{J}=0.35$ and $n_{x_{j}}=1 / 3$.

the capacitance matrix. To account for the fact that the total number of Cooper pairs on the ring is conserved, we define new coordinates

$$
\begin{gathered}
\hat{n}_{1}^{\prime}=\hat{n}_{1}, \quad \hat{n}_{2}^{\prime}=-\hat{n}_{2}, \quad \hat{n}_{3}^{\prime}=\hat{n}_{1}+\hat{n}_{2}+\hat{n}_{3}=n_{0}, \\
\hat{\phi}_{1}^{\prime}=\hat{\phi}_{1}-\hat{\phi}_{3}, \quad \hat{\phi}_{2}^{\prime}=\hat{\phi}_{3}-\hat{\phi}_{2}, \quad \hat{\phi}_{3}^{\prime}=\hat{\phi}_{3},
\end{gathered}
$$

where $n_{0}$ is the conserved total charge number, which is controlled by the external biases [20]. In the new coordinates, the Hamiltonian $\hat{H}_{\text {ring }}$ is

$$
\begin{aligned}
\hat{H}_{\text {ring }}= & E_{C_{\Sigma}}\left(\left(\hat{n}_{1}^{\prime}-\frac{1}{2}\left(n_{0}+n_{x_{1}}-n_{x_{3}}\right)\right)^{2}\right. \\
& \left.+\left(\hat{n}_{2}^{\prime}+\frac{1}{2}\left(n_{0}+n_{x_{2}}-n_{x_{3}}\right)\right)^{2}-\hat{n}_{1}^{\prime} \hat{n}_{2}^{\prime}\right) \\
& -E_{J}\left(\cos \left(\hat{\phi}_{1}^{\prime}-\frac{1}{3} \phi_{x}\right)+\cos \left(\hat{\phi}_{2}^{\prime}-\frac{1}{3} \phi_{x}\right)\right. \\
& \left.+\cos \left(\hat{\phi}_{1}^{\prime}+\hat{\phi}_{2}^{\prime}+\frac{1}{3} \phi_{x}\right)\right),
\end{aligned}
$$

where $E_{C_{\Sigma}}=(2 e)^{2} / C_{\Sigma}$ is the charging energy with $C_{\Sigma}=$ $3 C_{J}+C_{x}+C_{c}$.

In terms of the ring eigenbasis $\{|k\rangle ; k=0,1,2, \ldots\}$, we have

$$
\hat{H}_{\text {ring }}=\sum_{k>0} \omega_{k}|k\rangle\langle k|,
$$

where $\omega_{k}$ is the eigenenergy ${ }^{1}$ associated with the excited state $|k\rangle(k>0)$, and we have subtracted the ground-state energy of Eq. (4), so that $\omega_{0}=0$. Then $\omega_{k}$ represent ground-to-excitedstate transition frequencies that would be observed in spectra. In Fig. 1(b) we plot the first four excited-state energies $\omega_{k}$ $(k=1,2,3,4)$ as a function of the reduced external flux $\phi_{x}$.

\footnotetext{
${ }^{1}$ In this paper, we set $\hbar=1$.
} 
These eigenenergies are arranged in pairs; for large ranges of $\phi_{x}, \omega_{1}$ and $\omega_{2}$ are nearly degenerate and so are $\omega_{3}$ and $\omega_{4}$. Circulation of signals in the device is mediated by these excitations: depending on the external biases and the driving frequency, signals emitted from different excitations interfere constructively or destructively, resulting in clockwise or counterclockwise circulation $[5,19]$. This resembles the operation of a ferrite circulator where nonreciprocal transmission is created by interference of nearly degenerate, counterpropagating modes [4].

To compute output fields scattering from the circulator, we make use of the SLH framework [28,29]. We derive a Hamiltonian description of quantized bosonic fields for the waveguides interacting with the ring system. The total Hamiltonian for the combined system is (see Appendix A for derivation)

$$
\hat{H}_{\text {tot }}=\hat{H}_{\text {ring }}+\hat{H}_{\text {wg }}+\hat{H}_{\text {int }},
$$

where $\hat{H}_{\text {ring }}$ is given in Eq. (4) and the waveguide Hamiltonian $\hat{H}_{\text {wg }}$ is

$$
\hat{H}_{\mathrm{wg}}=\sum_{j=1}^{3} \int_{-\infty}^{\infty} d \omega \omega \hat{a}_{j}^{\dagger}(\omega) \hat{a}_{j}(\omega),
$$

which is the sum of three independent continua of harmonic oscillator modes. The interaction Hamiltonian $\hat{H}_{\text {int }}$, under the Markov and rotating-wave approximations, is $[19,34]$

$$
\hat{H}_{\mathrm{int}}=\sum_{j=1}^{3} \sqrt{\frac{\Gamma}{2 \pi}} \int_{-\infty}^{\infty} d \omega\left(\hat{a}_{j}^{\dagger}(\omega) \hat{q}_{j,-}+\hat{a}_{j}(\omega) \hat{q}_{j,+}\right),
$$

where $\hat{q}_{j,-} \equiv\left(\hat{q}_{j,+}\right)^{\dagger}=\sum_{k<\ell}\left\langle k\left|\hat{q}_{j}\right| \ell\right\rangle|k\rangle\langle\ell|$ is the upper triangularized part (in the ring eigenstate basis) of $\hat{q}_{j}$ [19], which is the coupling operator given in terms of the charge operators as

$$
\begin{aligned}
\hat{q}_{1} & =\hat{n}_{1}^{\prime}+n_{x_{1}}^{\prime} \\
& =\hat{n}_{1}+n_{x_{1}}^{\prime}, \\
\hat{q}_{2} & =-\hat{n}_{2}^{\prime}+n_{x_{2}}^{\prime} \\
& =\hat{n}_{2}+n_{x_{2}}^{\prime}, \\
\hat{q}_{3} & =-\hat{n}_{1}^{\prime}+\hat{n}_{2}^{\prime}+n_{x_{3}}^{\prime} \\
& =\hat{n}_{3}-n_{0}+n_{x_{3}}^{\prime} .
\end{aligned}
$$

Here $n_{x_{j}}^{\prime}$ are the rescaled charge biases

$$
\begin{aligned}
& n_{x_{1}}^{\prime}=c_{1}\left(n_{0}-n_{x_{2}}-n_{x_{3}}\right)-c_{2} n_{x_{1}}, \\
& n_{x_{2}}^{\prime}=c_{1}\left(n_{0}-n_{x_{1}}-n_{x_{3}}\right)-c_{2} n_{x_{2}}, \\
& n_{x_{3}}^{\prime}=c_{2}\left(n_{0}-n_{x_{3}}\right)-c_{1}\left(n_{x_{1}}+n_{x_{2}}\right),
\end{aligned}
$$

where $c_{1}=C_{J} /\left(C_{x}+C_{c}\right)$, and $c_{2}=\left(C_{J}+C_{x}+C_{c}\right) /\left(C_{x}+\right.$ $C_{c}$ ). In Eq. (8) $\Gamma$ is the waveguide-ring coupling strength explicitly given by [35-38]

$$
\Gamma=16 \frac{Z_{\mathrm{wg}}}{R_{K}}\left(\frac{C_{c}}{C_{\Sigma}}\right)^{2} \omega_{d}=32 \alpha \frac{Z_{\mathrm{wg}}}{Z_{\mathrm{vac}}}\left(\frac{C_{c}}{C_{\Sigma}}\right)^{2} \omega_{d},
$$

where $Z_{\mathrm{wg}}$ is the waveguide impedance, $R_{K}=h / e^{2} \approx$ $25.8 \mathrm{k} \Omega$ is the resistance quantum, $\alpha=Z_{\mathrm{vac}} /\left(2 R_{K}\right) \approx 1 / 137$ is the fine-structure constant with $Z_{\mathrm{vac}} \approx 377 \Omega$ the vacuum impedance, and $\omega_{d}$ is the driving frequency. As $C_{c} / C_{\Sigma}<1$ by definition, for the typical situation of $Z_{\mathrm{wg}}=50 \Omega$ one finds $Z_{\mathrm{wg}} / Z_{\mathrm{vac}} \approx 0.13$ and therefore $\Gamma<0.03 \omega_{d}$, justifying the approximations used to derive $\hat{H}_{\text {int }}$. This holds for $Z_{\mathrm{wg}} \lesssim Z_{\mathrm{vac}}$ but may not for high-impedance waveguides [39]. The coupling strength $\Gamma$ additionally (as shown later) sets the scale for resonance conditions and acceptable parameter imperfections in the circulator ring.

Using the above Hamiltonians and considering singlemode weak coherent fields at the input ports with the amplitudes $\beta_{j}$ and the frequency $\omega_{d}$, the SLH master equation for the circulator density operator $\rho$ is given by $[19,28,29]$

$$
\dot{\rho}=-i\left[\hat{H}_{\text {ring }}+\hat{H}_{\text {drive }}, \rho\right]+\sum_{j=1}^{3} \mathcal{D}\left[\hat{b}_{\text {out }, j}\right] \rho,
$$

where

$$
\begin{aligned}
& \hat{H}_{\text {drive }}=-\frac{i}{2} \sqrt{\Gamma} \sum_{j=1}^{3}\left(\beta_{j} e^{-i \omega_{d} t} \hat{q}_{j,+}-\text { H.c. }\right), \\
& \hat{b}_{\text {out }, j}=\beta_{j} e^{-i \omega_{d} t} \mathbb{1}+\sqrt{\Gamma} \hat{q}_{j,-},
\end{aligned}
$$

and $\mathcal{D}[\hat{\mathcal{O}}] \rho=\frac{1}{2}\left(2 \hat{\mathcal{O}} \rho \hat{\mathcal{O}}^{\dagger}-\rho \hat{\mathcal{O}}^{\dagger} \hat{\mathcal{O}}-\hat{\mathcal{O}}^{\dagger} \hat{\mathcal{O}} \rho\right)$. In Eq. (12), the commutation represents coherent evolution of the ring system plus the effect of dynamics induced from the external driving fields which is described by $\hat{H}_{\text {drive }}$ in Eq. (13), whereas the dissipation is due to couplings to the waveguides. Equation (14) represents the standard input-output relation [28,34] in which the output field is the sum of the input field and the field radiated from the ring system.

\section{SCATTERING MATRIX ELEMENTS}

We define the scattering matrix element $S_{i j}$ for transfer of signals from port $j$ to port $i$ as the ratio of the outgoing amplitude to the incoming one:

$$
S_{i j}=\frac{\left\langle\hat{b}_{\text {out }, i}\right\rangle}{\left\langle\hat{b}_{\mathrm{in}, j}\right\rangle},
$$

where $\langle\hat{\mathcal{O}}\rangle=\operatorname{Tr}(\hat{\mathcal{O}} \rho)$ denotes the expectation value of an operator $\hat{\mathcal{O}}$ with $\rho$ the circulator ring density operator. $S_{i j}$ can be computed numerically by solving $\rho$ using the master equation in Eq. (12). However, in this section we harness the adiabatic elimination technique [28,29], which allows us to express scattering of the open waveguide-ring system in terms of the isolated ring excitations, to derive a semianalytical expression for $S_{i j}$. This expression precisely describes the working principle of the circulator and helps to find the conditions to obtain optimal circulation.

\section{A. Adiabatic elimination}

When a quantum system can be decomposed into a fast subspace $\mathcal{F}$ and a slow subspace $\mathcal{S}$, we can adiabatically eliminate its fast dynamics and consider its slow dynamics only [28]. For the circulator ring system, its fast subspace consists of the excited states $\mathcal{F}=\{|k\rangle, k>0\}$, whereas its slow subspace contains the ground state only, $\mathcal{S}=\{|0\rangle\}$ [19]. In Appendix B we outline the calculations for performing the adiabatic elimination on the circulator system. We find the 
scattering matrix element $S_{i j}$ restricted to the slow subspace as

$$
S_{i j}=\delta_{i j}-\sum_{k>0} \frac{\left\langle k\left|\hat{q}_{j}\right| 0\right\rangle\left\langle 0\left|\hat{q}_{i}\right| k\right\rangle}{i \Delta \omega_{k} / \Gamma+\gamma_{k} / 2},
$$

where $\left\langle k\left|\hat{q}_{j}\right| 0\right\rangle$ is the excitation amplitude due to the coupling operator $\hat{q}_{j},\left\langle 0\left|\hat{q}_{i}\right| k\right\rangle$ is the relaxation amplitude due to the coupling operator $\hat{q}_{i}, \Delta \omega_{k}=\omega_{k}-\omega_{d}$ is the detuning of the excited eigenenergy $\omega_{k}$ from the driving frequency $\omega_{d}$, and $\gamma_{k}=\sum_{j=1}^{3}\left|\left\langle 0\left|\hat{q}_{j}\right| k\right\rangle\right|^{2}$ represents the total (dimensionless) decay rate of the excited state $|k\rangle$ due to waveguide couplings. Similar expressions to Eq. (16) can be found in related works $[20,40]$ but for different circulator systems and using different derivation methods. The delta function $\delta_{i j}$ in Eq. (16) is a consequence of the input-output relation in Eq. (14), in which the input field at one port contributes to the output field at that port, whereas the second term in Eq. (16) describes interference via the transient excitations of the circulator ring. Equation (16) demonstrates the importance of the external biases on signal scattering: they set the values of the matrix elements $\left\langle k\left|\hat{q}_{j}\right| 0\right\rangle$ as well as the transition energy $\omega_{k}$ (and subsequently the detuning $\Delta \omega_{k}$ ). Therefore, precise control over these biases is necessary to observe good circulation in the device.

At this point, it is instructional to consider the coherent power transmission of the scattered signals, $P_{j}=\sum_{i=1}^{3}\left|S_{i j}\right|^{2}$. Taking $\left|S_{i j}\right|^{2}$ in Eq. (16) and summing over $i$, we find that

$$
\begin{aligned}
P_{j}=\sum_{i=1}^{3}\left|S_{i j}\right|^{2}= & 1-\sum_{k>0} \frac{\left|\left\langle 0\left|\hat{q}_{j}\right| k\right\rangle\right|^{2} \gamma_{k}}{\left(\frac{i}{\Gamma} \Delta \omega_{k}+\frac{1}{2} \gamma_{k}\right)\left(-\frac{i}{\Gamma} \Delta \omega_{k}+\frac{1}{2} \gamma_{k}\right)} \\
& +\sum_{k, \ell>0} \frac{\left\langle k\left|\hat{q}_{j}\right| 0\right\rangle\left\langle 0\left|\hat{q}_{j}\right| \ell\right\rangle Q_{k \ell}}{\left(\frac{i}{\Gamma} \Delta \omega_{k}+\frac{1}{2} \gamma_{k}\right)\left(-\frac{i}{\Gamma} \Delta \omega_{\ell}+\frac{1}{2} \gamma_{\ell}\right)},
\end{aligned}
$$

where $Q_{k \ell}=\sum_{j=1}^{3}\left\langle 0\left|\hat{q}_{j}\right| k\right\rangle\left\langle\ell\left|\hat{q}_{j}\right| 0\right\rangle$ with $k, \ell>0$. Numerically, we observe that $\left|Q_{k \neq \ell}\right| \ll\left|Q_{k k}\right| \equiv \gamma_{k}$ (see Fig. 11 in Appendix C). Hence, in the second line of Eq. (17) we can ignore terms with $k \neq \ell$ and consider only those with $k=\ell$. We find that

$$
P_{j}=\sum_{i=1}^{3}\left|S_{i j}\right|^{2}=1,
$$

which merely reflects the energy conservation constraint. We note that if incoherent scattering occurs (due to dephasing, etc.) then the coherent power transfer condition relaxes to $P_{j}<1$; i.e., scattering into incoherent channels would appear as loss of total power in the coherent subspace.

\section{B. Conditions for optimal circulation}

Based on the results in the previous section, we deduce the conditions for achieving optimal clockwise circulation. Note that the conditions for optimal counterclockwise circulation can be found in a similar manner. We first introduce the scattering matrix for ideal (clockwise) circulation,

$$
S_{\text {ideal }}=\left(\begin{array}{lll}
0 & 1 & 0 \\
0 & 0 & 1 \\
1 & 0 & 0
\end{array}\right),
$$
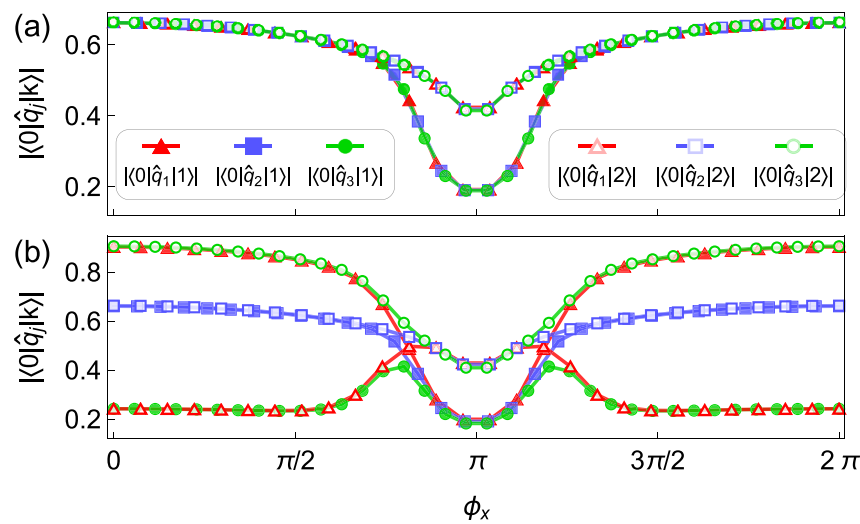

FIG. 2. Magnitudes of the coupling matrix elements $\left|\left\langle 0\left|\hat{q}_{j}\right| 1\right\rangle\right|$ (solid markers) and $\left|\left\langle 0\left|\hat{q}_{j}\right| 2\right\rangle\right|$ (open markers) with $j=1,2,3$ as functions of the reduced external flux $\phi_{x}$ when biasing the three ring islands identically at charge biases of $1 / 3$ for (a) a symmetric circulator ring with $E_{J_{j}}=E_{J}$ and $E_{C_{\Sigma}} / E_{J}=0.35$ and (b) an asymmetric circulator ring with $E_{J_{1}} / E_{J}=1, E_{J_{2}} / E_{J}=1.01, E_{J_{3}} / E_{J}=0.99$, and $E_{C_{\Sigma}} / E_{J}=0.35$.

noting that we are indifferent to the output phases of the nonzero elements. Since a diagonal element $S_{j j}$ from Eq. (16) is given by

$$
S_{j j}=1-\sum_{k>0} \frac{\left|\left\langle 0\left|\hat{q}_{j}\right| k\right\rangle\right|^{2}}{i \Delta \omega_{k} / \Gamma+\gamma_{k} / 2},
$$

to have $S_{11}=S_{22}=S_{33}$ one needs

$$
\left|\left\langle 0\left|\hat{q}_{1}\right| k\right\rangle\right|=\left|\left\langle 0\left|\hat{q}_{2}\right| k\right\rangle\right|=\left|\left\langle 0\left|\hat{q}_{3}\right| k\right\rangle\right| \text { for } k>0 .
$$

From Eq. (9) we have $\left|\left\langle 0\left|\hat{q}_{j}\right| k\right\rangle\right|=\left|\left\langle 0\left|\hat{n}_{j}\right| k\right\rangle\right|$ with $\hat{n}_{j}$ the original charge operator on the island $j$, so the above condition is equivalent to $\left|\left\langle 0\left|\hat{n}_{1}\right| k\right\rangle\right|=\left|\left\langle 0\left|\hat{n}_{2}\right| k\right\rangle\right|=\left|\left\langle 0\left|\hat{n}_{3}\right| k\right\rangle\right|$, suggesting that the three islands of the circulator ring should be symmetric. ${ }^{2}$ In the case of a symmetric circulator ring with identical Josephson junctions, this implies that the charge biases on the islands should also be identical. In Fig. 2 we plot $\left|\left\langle 0\left|\hat{q}_{j}\right| 1\right\rangle\right|$ (solid markers) and $\left|\left\langle 0\left|\hat{q}_{j}\right| 2\right\rangle\right|$ (open markers) with $j=1,2,3$ versus the reduced external flux $\phi_{x}$ for both symmetric [Fig. 2(a)] and asymmetric [Fig. 2(b)] circulator rings at identical charge biases $n_{x_{j}}$ of $1 / 3$. For a symmetric ring, we observe in Fig. 2(a) that the condition in Eq. (20) is satisfied for the whole range of $\phi_{x}$ from zero to $2 \pi$. For an asymmetric ring with different Josephson energies in Fig. 2(b), the condition in Eq. (20) is approximately met for a small interval around $\phi_{x}=\pi$.

From Eq. (9), we have $\hat{q}_{3}=-\hat{q}_{1}-\hat{q}_{2}+\sum_{j=1}^{3} n_{x_{i}}^{\prime}$. By this we can recast the condition in Eq. (20) to $\left|\left\langle 0\left|\hat{q}_{1}\right| k\right\rangle\right|=$ $\left|\left\langle 0\left|\hat{q}_{2}\right| k\right\rangle\right|=\left|\left\langle 0\left|\hat{q}_{1}\right| k\right\rangle+\left\langle 0\left|\hat{q}_{2}\right| k\right\rangle\right|$. This is then translated into

\footnotetext{
${ }^{2}$ When the islands of the ring circulator are symmetric, its Hamiltonian is invariant with respect to cyclic permutations of the node labels, $j=1 \rightarrow 2 \rightarrow 3 \rightarrow 1$ or $j=1 \rightarrow 3 \rightarrow 2 \rightarrow 1$. Under these permutations, $\hat{n}_{j}$ becomes $\hat{n}_{j^{\prime}}$, the ground state $|0\rangle$ is unchanged, and the excited state $|k\rangle$ picks up a phase, which results in $\left|\left\langle 0\left|\hat{n}_{j}\right| k\right\rangle\right|=$ $\left|\left\langle 0\left|\hat{n}_{j^{\prime}}\right| k\right\rangle\right|$.
} 
the following conditions:

$$
\begin{aligned}
\left\langle 0\left|\hat{q}_{j}\right| k\right\rangle & =r_{k} e^{i \varphi_{j, k}} \quad \text { for } j=1,2, \\
\left|\varphi_{1, k}-\varphi_{2, k}\right| & =\frac{2 \pi}{3} \quad \text { for } k>0 .
\end{aligned}
$$

The former condition tells us that the matrix elements between the ground state $|0\rangle$ and the excitation $|k\rangle$ of the coupling operators $\hat{q}_{1}$ and $\hat{q}_{2}$ should have the same magnitude, while the latter imposes a specific constraint on the phases of these matrix elements. These two conditions were pointed out in Ref. [20] based on a system that includes external cavities on the output of each circulator waveguide, whereas our analysis is based directly on the circulated scattering elements.

We next derive the relations between the driving frequency $\omega_{d}$, the coupling strength $\Gamma$, and the first two transition energies $\omega_{1}$ and $\omega_{2}$ to observe optimal circulation. We notice that the strong anharmonicity of the circulator ring [see Fig. 1(b)] allows us to consider contributions of only the first two excitations to signal circulation and ignore those of higher excitations; thus, in Eq. (16) the values of $k$ are truncated to $\{1,2\}$. We define new parameters

$$
x_{k}=\frac{\left|r_{k}\right|^{2}}{\left(\left(\Delta \omega_{k} / \Gamma\right)^{2}+\left(\gamma_{k} / 2\right)^{2}\right)^{1 / 2}}, \quad \tan \left(\theta_{k}\right)=\frac{-2 \Delta \omega_{k}}{\Gamma \gamma_{k}},
$$

for $k=1$, 2. From Eqs. (21) and (22) we recast $S_{i j}$ in terms of $r_{k}$ and $\varphi_{j, k}$ and subsequently $x_{k}$ and $\theta_{k}$. For example, we find $S_{11}=1-x_{1} e^{i \theta_{1}}-x_{2} e^{i \theta_{2}}$ and $S_{21}=x_{1} e^{i\left(\theta_{1} \pm_{1} 2 \pi / 3\right)}+$ $x_{2} e^{i\left(\theta_{2} \pm_{2} 2 \pi / 3\right)}$, where the signs $\pm_{k}$ can be different between the levels [20]. Using Eq. (18), the first column of the ideal scattering matrix $\left(S_{11}, S_{21}, S_{31}\right)=(0,0,1)$ is equivalent to $\left(S_{11}, S_{21}\right)=(0,0)$, yielding

$$
\begin{aligned}
& S_{11}=1-x_{1} e^{i \theta_{1}}-x_{2} e^{i \theta_{2}}=0, \\
& S_{21}=x_{1} e^{i\left(\theta_{1}-2 \pi / 3\right)}+x_{2} e^{i\left(\theta_{2}+2 \pi / 3\right)}=0,
\end{aligned}
$$

where we have chosen specifically the sign of $\pm_{k}$ in the phase factors of $S_{21}$. The solution for this system of equations is

$$
x_{1}=x_{2}=1 / \sqrt{3}, \quad \theta_{1}=-\theta_{2}=\pi / 6,
$$

which results in

$$
-\Delta \omega_{1}=\frac{1}{2 \sqrt{3}} \gamma_{1} \Gamma, \quad \Delta \omega_{2}=\frac{1}{2 \sqrt{3}} \gamma_{2} \Gamma .
$$

We recall that $\gamma_{1}$ and $\gamma_{2}$ respectively represent the decay rates of the first two excited states $|1\rangle$ and $|2\rangle$. We aim to operate the circulator at the parameter ranges such that the two excited states are nearly degenerate, so we can have $\gamma_{1} \simeq \gamma_{2}=\gamma$. This, combining with the results in Eq. (27), yields

$$
\begin{aligned}
\omega_{d} & \simeq \frac{1}{2}\left(\omega_{1}+\omega_{2}\right), \\
\Gamma & \simeq \frac{\sqrt{3}}{\gamma}\left(\omega_{2}-\omega_{1}\right) .
\end{aligned}
$$

The former condition ensures that the driving fields excite the first two nearly degenerate excited states equally. Meanwhile, the latter introduces a concrete relation between the coupling strength $\Gamma$ and the eigenenergy difference $\omega_{2}-\omega_{1}$ [40], which can be met by suitably tuning the reduced external flux $\phi_{x}$. Note that the same results in Eqs. (26)-(29) are obtained when using either the second column or the third column of $S_{\text {ideal }}$.

Based on the above conditions, we implement a simple numerical scheme to compute the optimal parameters for circulation. Considering a symmetric Josephson-junction ring with identical Josephson energies, the condition in Eq. (20) indicates that we should choose identical charge biases (for example, at $1 / 3$ of a Cooper pair), while the driving frequency $\omega_{d}$ should be chosen to be $\left(\omega_{1}+\omega_{2}\right) / 2$ as suggested by the condition in Eq. (28). The external flux $\phi_{x}$ is determined via the condition in Eq. (29). Noting that the charge offsets are already fixed $\left(n_{x_{j}}=1 / 3\right)$, the transitions $\omega_{1}$ and $\omega_{2}$, the decay rate $\gamma$, and the coupling $\Gamma$ are implicitly functions of $\phi_{x}$. Then the optimal value for $\phi_{x}$ is numerically found from the equation $\Gamma\left(\phi_{x}\right)=\sqrt{3}\left(\omega_{2}\left(\phi_{x}\right)-\omega_{1}\left(\phi_{x}\right)\right) / \gamma\left(\phi_{x}\right)$. For an asymmetric ring with different Josephson energies, it is no longer straightforward to estimate the optimal charge biases analytically. However, we can consider the relevant quantities as functions of the charge biases $n_{x_{j}}$ and the external flux $\phi_{x} \cdot{ }^{3} \mathrm{We}$ evaluate the optimal working point by numerically finding $n_{x_{j}}$ and $\phi_{x}$ that satisfy the conditions in Eqs. (20), (28), and (29).

\section{OPTIMIZATION OF OPERATING PARAMETERS}

We note that solving the conditions in Eqs. (20), (28), and (29) gives physical insights into the optimal working parameters above. However, extracting the quantities such as $\omega_{1}, \omega_{2}$, and $\Gamma$ from experiments to sufficiently high accuracy may be difficult in practice. Therefore, we now implement an optimization procedure that finds the optimal working points using a standard optimization method. We have checked that this approach gives the same result for $\phi_{x}, n_{x_{j}}$, and $\omega_{d}$ as solving Eqs. (20), (28), and (29), as described in the previous section.

We optimize a cost function that finds points of high fidelity $F\left(|S|, S_{\text {ideal }}\right)$ [41] between the computed scattering matrix $S$ and the ideal clockwise scattering matrix, $S_{\text {ideal }}$, in Eq. (19). We present the optimization results for both symmetric and asymmetric circulator rings.

We define the fidelity $F(A, B)$ between two matrices $A$ and $B$ as

$$
F(A, B)=1-\frac{1}{\|A\|\|B\|}\left(\sum_{i, j}|A(i, j)-B(i, j)|^{2}\right)^{1 / 2},
$$

where $\|X\|=\sqrt{\operatorname{Tr}\left(X X^{\dagger}\right)}$ denotes the norm of a matrix $X$. In Eq. (30) the second term describes a distance measure between two matrices. The fidelity is thus complementary to the distance measure: if two matrices are very similar to each other, their distance measure will be close to zero but their fidelity will be close to one. It is worth noting that a noncirculating device with $S \simeq \mathbb{1}$ and a counterclockwise-circulating one with $S \simeq S_{\text {ideal }}^{\top}$ have $F\left(\mathbb{1}, S_{\text {ideal }}\right)=F\left(S_{\text {ideal }}^{\top}, S_{\text {ideal }}\right) \approx 0.18$, which sets the neutral value of the fidelity.

We fix the energy scales of the circulator (i.e., the Josephson energies $E_{J_{j}}$ and the charging energy $E_{C_{\Sigma}}$ ) and employ a

\footnotetext{
${ }^{3} \mathrm{As} \Gamma$ is given in terms of $\omega_{d}$ as in Eq. (11) and $\omega_{d}$ is chosen to be $\left(\omega_{1}+\omega_{2}\right) / 2$ depending on $\phi_{x}, \Gamma$ is treated as a function of $\phi_{x}$.
} 
standard optimization routine (FindMaximum in MATHEMATICA) to optimize the fidelity over five control parameters, namely, the driving frequency $\omega_{d}$, the reduced external flux $\phi_{x}$, and the three charge biases $n_{x_{1}}, n_{x_{2}}$, and $n_{x_{3}}$. Over the course of optimization we also track the variations of other quantities, including the coupling matrix elements $\left\langle 0\left|\hat{q}_{j}\right| k\right\rangle$, the coupling strength $\Gamma$, and the ring eigenenergies $\omega_{1}$ and $\omega_{2}$. We show that the optimization converges relatively fast after less than 50 optimization steps. In a realistic experiment, this would require sequential measurements of the full scattering matrix. Given typical experimental time per single scattering matrix measurement of 10-100 $\mu$ s [42], the total optimization would take $0.5-5 \mathrm{~ms}$, indicating feasibly fast calibration of the device.

\section{A. Symmetric Josephson-junction ring}

We consider a symmetric Josephson-junction ring with $E_{J_{j}}=E_{J}(j=1,2,3), E_{C_{\Sigma}} / E_{J}=0.35$, and $\Gamma / E_{J} \approx 0.0025$ for $Z_{\mathrm{wg}}=50 \Omega$ and $\omega_{d} / E_{J}=0.8$ and perform five optimizations for the fidelity $F\left(\left|S_{\text {sym }}\right|, S_{\text {ideal }}\right)$. In Appendix D we show specific parameter values for simulations. Here the ratio $E_{C_{\Sigma}} / E_{J}=0.35$ is chosen to be in between the "Cooper-pairbox" $\left(E_{C_{\Sigma}} / E_{J} \gg 1\right)$ and "transmon" $\left(E_{C_{\Sigma}} / E_{J} \ll 1\right)$ regimes for the following reasons. First, operating the device outside the Cooper-pair-box regime avoids charge sensitivity. Second, as pointed out in Ref. [20], reducing $E_{C_{\Sigma}} / E_{J}$ into the transmon regime, which intuitively should make the device insensitive to charge noise, actually destroys the circulation feature. This is because in this regime all the coupling matrix elements can be chosen to be purely imaginary, breaking down the interference effect [see Eq. (16)]. Third, we find that when decreasing $E_{C_{\Sigma}} / E_{J}$ the decay rate $\gamma$ in Eq. (29) increases while the transition difference $\omega_{2}-\omega_{1}$ decreases. Thus, reducing $E_{C_{\Sigma}} / E_{J}$ results in a small optimal coupling strength $\Gamma$ [which is proportional to $\left(\omega_{2}-\omega_{1}\right) / \gamma$ as in Eq. (29)] as well as a small working bandwidth. We confirm these numerically in Appendix D. Additionally, as shown later a small $\Gamma$ will put hard constraints on junction fabrication.

Each of the optimizations is initialized with a set of external parameters chosen randomly within certain ranges. That is, $\omega_{d} / E_{J} \in[0.70,0.85], \phi_{x} \in[1.00,2.14]$, and $n_{x_{j}} \in[0,1]$, reflecting the experimental uncertainties in initial parameters immediately after cooldown of the device, e.g., due to charge frozen in the substrate materials, flux defects, and charge-reset noise $\delta Q \sim \sqrt{k_{B} T C}$ worth approximately one electron for $\mathrm{fF}$ gate capacitors at the cooling temperature $T \sim 1 \mathrm{~K}[43,44]$. The ranges of $\omega_{d}$ and $\phi_{x}$ are intentionally selected such that $\omega_{2}-\omega_{1}$ is neither too large nor too small compared to $\Gamma$, as suggested from Eq. (29) and observed from the circulator spectrum in Fig. 1(b). We track the fidelity during optimization steps to see how quickly the optimization proceeds. We also plot the optimization process for a representative selection of randomly initialized external control parameters in Appendix E.

As shown in Fig. 3 four out of five example optimizations yield a very high fidelity $(\approx 1)$, after 25 to 30 optimization steps. In these cases the driving frequency $\omega_{d}$ and the reduced flux $\phi_{x}$ in Figs. 13(a) and 13(b) in Appendix E evolve to well-defined values at about $0.82 E_{J}$ and 1.77 , respectively.

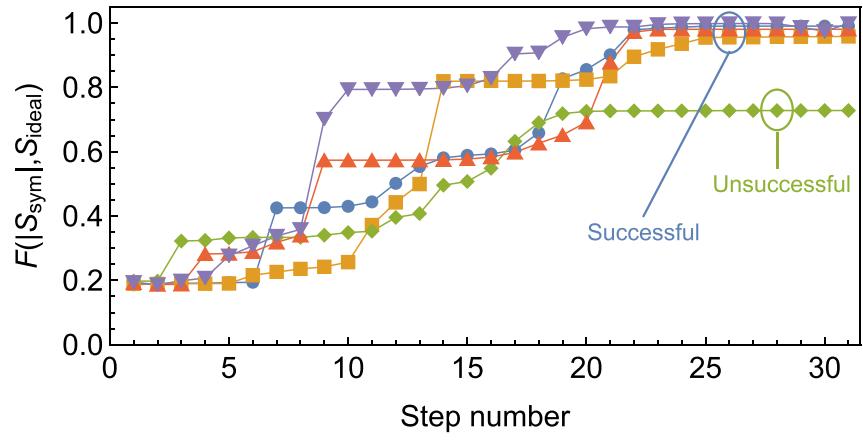

FIG. 3. Examples of optimization for a symmetric circulator ring. We optimize the fidelity $F\left(\left|S_{\text {sym }}\right|, S_{\text {ideal }}\right)$ between the numerically computed scattering matrix $S_{\text {sym }}$ with respect to the ideal (clockwise) one $S_{\text {ideal }}$ for five times. Each optimization begins with a different set of initial external parameters chosen randomly from specific ranges (see also main text) and takes 30 steps to complete. Relevant parameters are chosen as $E_{J j}=E_{J}, E_{C_{\Sigma}} / E_{J}=0.35$, and $\Gamma / E_{J} \approx 0.0025$ for $Z_{\mathrm{wg}}=50 \Omega$ and $\omega_{d} / E_{J}=0.8$.

Meanwhile, the three charge biases $n_{x_{j}}$ in Figs. 13(c)-13(e) tend towards the same value with two apparent clusters near 0.4 .

The resulting power transfer matrix after the successful optimizations is

$$
\left|S_{\mathrm{opt}}\right|^{2} \approx\left(\begin{array}{ccc}
0.003 & 0.995 & 0.002 \\
0.002 & 0.003 & 0.995 \\
0.995 & 0.002 & 0.003
\end{array}\right)
$$

This corresponds to insertion loss of $\mathrm{IL} \approx 0.02 \mathrm{~dB}$ while the reflection and the isolation are $\mathrm{R} \approx-25 \mathrm{~dB}$ and $\mathrm{IS} \approx$ $-27 \mathrm{~dB}$, respectively, where

$$
\begin{aligned}
\mathrm{IL} & =10 \log _{10}\left(\left(\left|S_{12}\right|^{2}+\left|S_{23}\right|^{2}+\left|S_{31}\right|^{2}\right) / 3\right), \\
\mathrm{R} & =10 \log _{10}\left(\left(\left|S_{11}\right|^{2}+\left|S_{22}\right|^{2}+\left|S_{33}\right|^{2}\right) / 3\right), \\
\mathrm{IS} & =10 \log _{10}\left(\left(\left|S_{13}\right|^{2}+\left|S_{21}\right|^{2}+\left|S_{32}\right|^{2}\right) / 3\right) .
\end{aligned}
$$

In some cases the optimization can be trapped in a suboptimal configuration; for example, the unsuccessful optimization (solid green diamond) in Fig. 3 yields a substantially reduced fidelity $(\approx 0.7)$. In this scenario the three charge biases, as shown in Figs. 13(c)-13(e), arrive at rather different final values, partly explaining why the fidelity for that optimization is not as high as for the other optimizations. This failure, possibly, is due to our use of a very simple optimization algorithm and may be circumvented by repeating the optimization from a different starting parameter set or by employing more sophisticated parameter optimization routines.

Furthermore, we observe that after optimization the condition in Eq. (20) is typically satisfied. This is demonstrated in Figs. 14(a)-14(f) in Appendix E which show that the matrix element magnitudes $\left|\left\langle 0\left|\hat{q}_{j}\right| k\right\rangle\right|$ for $j=1,2,3$ and $k=1,2$ approach the same value for the successful optimizations. We also confirm the conditions in Eqs. (28) and (29) by plotting the two ratios $2 \omega_{d} /\left(\omega_{1}+\omega_{2}\right)$ and $\gamma \Gamma /\left(\omega_{2}-\omega_{1}\right)$ respectively in Figs. 14(g) and 14(h). 


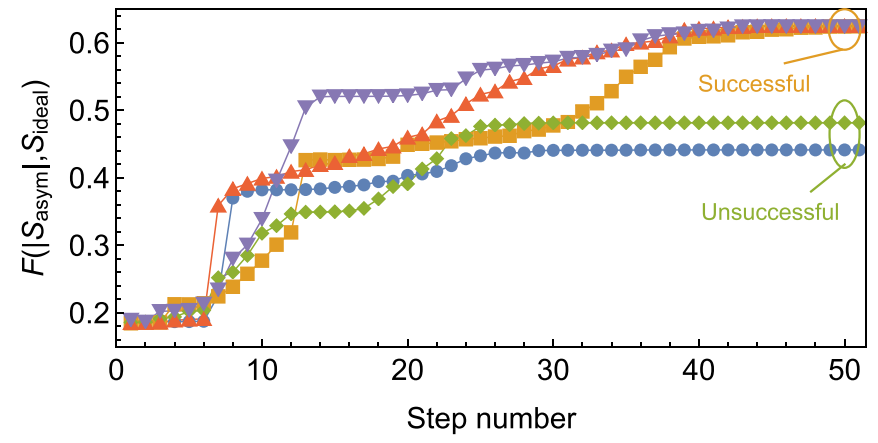

FIG. 4. Similar to Fig. 3 but for an asymmetric circulator ring. Relevant parameters are chosen as $E_{J_{1}} / E_{J}=1, E_{J_{2}} / E_{J}=1.01$, $E_{J_{3}} / E_{J}=0.99, E_{C_{\Sigma}} / E_{J}=0.35$, and $\Gamma / E_{J} \approx 0.0025$ for $Z_{\mathrm{wg}}=50 \Omega$ and $\omega_{d} / E_{J}=0.8$.

\section{B. Asymmetric Josephson-junction ring}

Realistic device fabrication is always imperfect, giving rise to junction asymmetry. Therefore, we introduce asymmetry in the circulator junctions as $E_{J_{1}}=E_{J}, E_{J_{2}}=E_{J}+$ $\delta E_{J_{2}}$, and $E_{J_{3}}=E_{J}+\delta E_{J_{3}}$. To illustrate the effect of imperfect Josephson junctions we choose $\delta E_{J_{2}} / E_{J}=0.01$ and $\delta E_{J_{3}} / E_{J}=-0.01$ and show the corresponding optimization results in Fig. 4. Such junction asymmetry is plausible in realistic experiments [45,46]. For example, Ref. [46] reported fabrication of on-chip Josephson junctions with high reproducibility and normal resistance $\left(R_{N}\right)$ variation as small as $1.2 \%$ which corresponds to $\left|\delta E_{J}\right| / E_{J} \sim 0.012$. $^{4}$

In Fig. 4 three out of five optimizations converge to fidelities just above 0.6 after 50 steps of optimization. The power transfer matrix for these optimizations is

$$
\left|S_{\text {opt }}\right|^{2} \approx\left(\begin{array}{lll}
0.08 & 0.70 & 0.22 \\
0.35 & 0.06 & 0.59 \\
0.57 & 0.24 & 0.19
\end{array}\right)
$$

showing that the device circulates imperfectly with $\mathrm{IL} \approx$ $-2.1 \mathrm{~dB}, \mathrm{R} \approx-9.5 \mathrm{~dB}$, and $\mathrm{IS} \approx-5.7 \mathrm{~dB}$.

Similar to the symmetric case, the driving frequency $\omega_{d}$ and the reduced flux $\phi_{x}$ in Figs. 15(a) and 15(b) of Appendix E approach well-defined values at about $0.70 E_{J}$ and 2.41 , respectively. In contrast to the symmetric case, the three charge biases in Figs. 15(c)-15(e) tend towards different values during optimizations. However, as shown in Fig. 16 the conditions in Eqs. (20) and (28) are still approximately fulfilled: the matrix element magnitudes $\left|\left\langle 0\left|\hat{q}_{j}\right| k\right\rangle\right|(j=1,2,3$ and $k=1,2)$ are quite close to each other [see Figs. 16(a)16 (f)] and the ratio $2 \omega_{d} /\left(\omega_{1}+\omega_{2}\right)$ gets to almost exactly 1 [see Fig. 16(g)]. The ratio $\gamma \Gamma /\left(\omega_{2}-\omega_{1}\right)$ in Fig. 16(h) approaches about 0.45 , far below the optimal value $\sqrt{3}$ required in the condition in Eq. (29).

Comparing the fidelities in Figs. 3 and 4, we observe a $40 \%$ reduction in the optimized fidelity as a result of only $1 \%$ asymmetry in the ring junctions. This follows from the fact

${ }^{4}$ From the Ambegaokar-Baratoff relation [46] $E_{J}=A\left(\bar{R}_{N}+\right.$ $\left.\delta R_{N}\right)^{-1}=\bar{E}_{J}+\delta E_{J}$, where $A$ is a constant and $\bar{E}_{J}=A \bar{R}_{N}^{-1}$, we can estimate $\left|\delta E_{J}\right| / \bar{E}_{J}=\left|\delta R_{N}\right| / \bar{R}_{N}$.
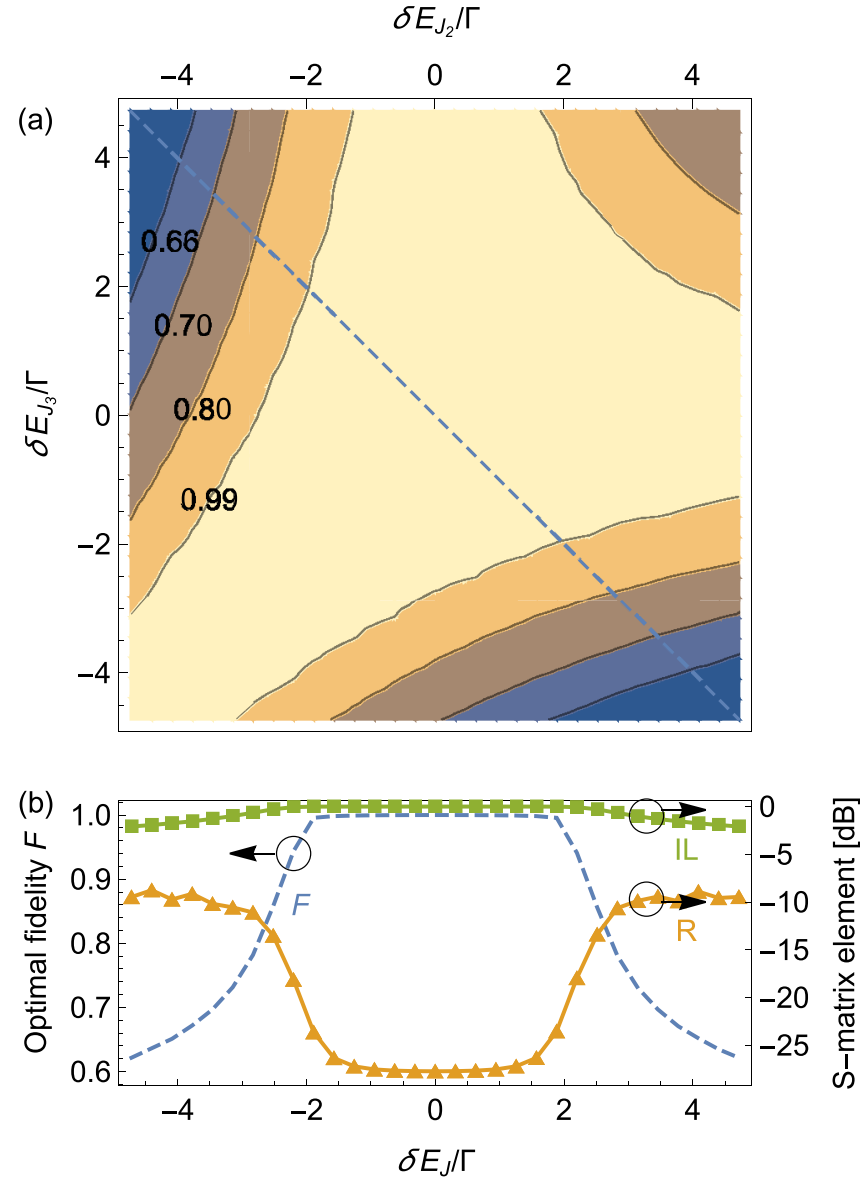

FIG. 5. (a) Contour plot of the optimal fidelity, $F$, versus the ratios of the junction disorders $\delta E_{J_{2}}$ and $\delta E_{J_{3}}$ to the coupling strength $\Gamma$. (b) Diagonal cut of the optimal fidelity in (a) (blue dashed line) which corresponds to junction asymmetries $\delta E_{J_{2}}=-\delta E_{J_{3}}=\delta E_{J}$ considered in Fig. 4. Also shown in (b) includes reflection, R, and insertion loss, IL. To generate the plots, we introduce junction asymmetry as $E_{J_{1}}=E_{J}, E_{J_{2}}=E_{J}+\delta E_{J_{2}}$, and $E_{J_{3}}=E_{J}+\delta E_{J_{3}}$ and for each pair $\left(\delta E_{J_{2}}, \delta E_{J_{3}}\right)$ we find the optimal fidelity by performing optimization over the external control parameters and calculate the scattering matrix elements at the optimized working points. Here at the leftmost or rightmost of (b), $\left|\delta E_{J}\right| / \Gamma \approx 4.7$ and the optimal fidelity is $F \approx 0.6$, consistent with the optimized value in Fig. 4.

that in this asymmetric case $\Gamma\left(\approx 0.0025 E_{J}\right)$ is substantially smaller than the detuning $\omega_{1}-\omega_{2}\left(\approx 0.01 E_{J}\right)$ between the two excited states. Thus, there is no driving frequency that simultaneously couples strongly to both states $|1\rangle$ and $|2\rangle$, and subsequently the condition for interference between these states is inhibited. To show that $\Gamma$ sets the tolerance level for asymmetries in junction parameters, in Fig. 5(a) we plot the optimal fidelity versus the two ratios $\delta E_{J_{2}} / \Gamma$ and $\delta E_{J_{3}} / \Gamma$ and in Fig. 5(b) we consider the example shown in Fig. 4 with $\delta E_{J_{2}}=-\delta E_{J_{3}}=\delta E_{J}$. We see in Figs. 5(a) and 5(b) that the optimal fidelity remains close to 1 for $\left|\delta E_{J}\right| / \Gamma$ as large as 2 but decreases quite quickly for larger $\left|\delta E_{J}\right| / \Gamma$. Accordingly, in Fig. 5(b) the reflection is below $-20 \mathrm{~dB}$ and the insertion loss is very close to $0 \mathrm{~dB}$ for that range of $\left|\delta E_{J}\right|$. The parameters used for the optimizations in Fig. 4 give $\delta E_{J} / \Gamma \approx 4.7$ corresponding to an optimal fidelity slightly above 0.6 , which 
(a)

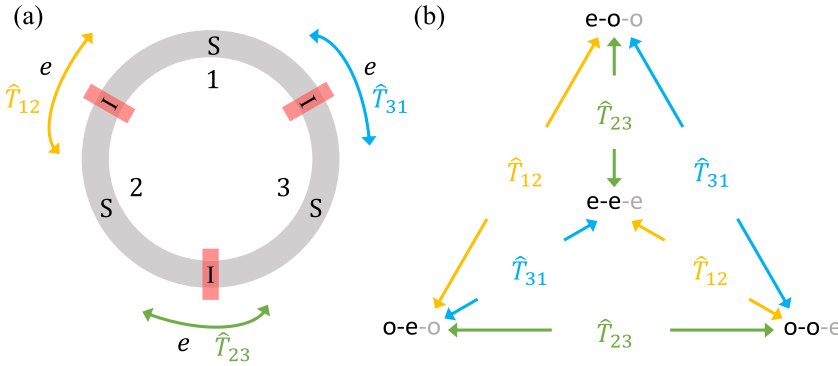

FIG. 6. (a) Quasiparticle tunneling in the circulator ring. The device comprises three superconducting-insulator-superconducting (SIS) junctions across which unpaired quasiparticles can tunnel. (b) Charge-parity sectors $\{e-e(-e), e-0(-0), o-e(-o), o-o(-e)\}$ assuming the total charge parity is even and tunneling operators $\hat{T}_{12}=$ $\sin \left(\left(\hat{\phi}_{1}^{\prime}+\hat{\phi}_{2}^{\prime}\right) / 2\right), \hat{T}_{23}=\sin \left(\hat{\phi}_{2}^{\prime} / 2\right)$, and $\hat{T}_{31}=\sin \left(\hat{\phi}_{1}^{\prime} / 2\right)$ connecting them (see Appendix F for derivation of these operators).

is consistent with the values at the leftmost or rightmost of Fig. 5(b).

\section{QUASIPARTICLES}

In the previous sections, we have specified values for the external control parameters that optimize the circulator performance. These parameters are subject to fluctuations due to voltage noise of various sources. Fast charge fluctuations with magnitudes much smaller than one electron have been studied in Ref. [19], while charge drifts comparable to one electron are expected to occur at a timescale much longer than the optimization time. Hence, in what follows we focus on quasiparticle formation and migration in the superconducting islands forming the circulator, which is a frequently encountered noise source in superconducting devices [47]. We analyze the effects of quasiparticles on the circulator performance. We anticipate quasiparticle formation will be slow relative to the internal dynamical timescales of the circulator, so our analysis is quasistatic. Quasiparticle tunneling causes large changes in the effective bias voltages, on the scale of half a Cooper pair, and thus is nonperturbative. We show that quasiparticle tunneling in the ring circulator results in several operating sectors characterized by the parity of charges on the islands. These sectors have different energy spectra and scatter signals differently, yielding different circulation performances. Spectroscopic measurements performed on the circulator ring over experimental timescales much larger than the quasiparticle formation and tunneling rates will show a mixture of the spectra from the different quasiparticle sectors.

\section{A. Parity-charge sectors}

As illustrated in Fig. 6(a) the circulator ring is in effect a loop of three superconducting-insulator-superconducting (SIS) junctions. Quasiparticles can tunnel across these junctions, giving rise to switching of parities of the electron numbers in the superconducting islands $[48,49]$. Since the ring is capacitively isolated from outside environments, the total number of electrons is conserved. The charge-parity configuration of the circulator ring can thus be represented by the parities of two out of the three islands, say, islands 1 and 2 only, which due to charge conservation determine the parity of the third island. Furthermore, in the following we assume the total charge parity of the three islands is even. Similar arguments hold for the case of an odd total charge parity, which is considered in Appendix G.

The above arguments yield four accessible charge-parity sectors which we label as e-e-e, e-0-0, o-e-o, and o-o-e, where e denotes even charge parity on the relevant island, and $\mathrm{o}$ denotes odd charge parity. Because of total charge-parity conservation, the label for the third island is redundant, so for brevity we drop this label. For example, e-o-o and e-o refer to the same charge parity sector, which includes all of the charge states satisfying $n_{1}^{\prime} \bmod 2=0$ and $n_{2}^{\prime} \bmod 2=1$ with $n_{1}^{\prime}$ and $n_{2}^{\prime}$ respectively the eigenvalues of the charge operators $\hat{n}_{1}^{\prime}$ and $\hat{n}_{2}^{\prime}{ }^{5}$ Similar definitions hold for e-e, o-e, and o-o.

The sectors are coupled to each other by tunneling of a quasiparticle between the adjacent islands. For example, coupling between the sectors e-e and e-o is via tunneling of a quasiparticle between islands 2 and 3. This is represented by the operator $\hat{T}_{23}=\sin \left(\left(\hat{\phi}_{3}-\hat{\phi}_{2}\right) / 2\right) \equiv \sin \left(\hat{\phi}_{2}^{\prime} / 2\right)[50,51]$ (see Appendix F for derivation). In Fig. 6(b) we illustrate all the quasiparticle-tunneling operators coupling among the four sectors.

Tunneling of a quasiparticle into or out of a superconducting island is equivalent to shifting the charge bias on that island by $\pm 1 e$ [25-27], i.e., by half a unit charge. For example, if the ring is initially in the e-e charge sector, with charge biases $\left(n_{x_{1}}, n_{x_{2}}, n_{x_{3}}\right)$, then tunneling of a quasiparticle from island 2 to 3 will leave the ring in the sector e-o with effective charge biases $\left(n_{x_{1}}, n_{x_{2}}-\frac{1}{2}, n_{x_{3}}+\frac{1}{2}\right)$. That is, quasiparticle tunneling changes the charge state and therefore the effective charge bias of the islands.

This property allows us to express the Hamiltonian for all charge sectors in a self-consistent form. To do so, we order the charge basis to group states within each of the charge-parity sectors, and in this ordered basis, the Hamiltonian matrix is block diagonal. Each sub-block of the Hamiltonian matrix is then given by a common functional form, $\underline{H}^{\text {ref }}\left(n_{x_{1}}, n_{x_{2}}, n_{x_{3}}\right)$, where the double underline denotes a matrix representation of an operator expressed in the charge basis:

$$
\begin{aligned}
& \underline{\underline{H}}_{\text {ring }}^{\mathrm{e}-\mathrm{e}}=\underline{\underline{H}}^{\mathrm{ref}}\left(n_{x_{1}}, n_{x_{2}}, n_{x_{3}}\right), \\
& \underline{\underline{H}}_{\text {ring }}^{\mathrm{e}-\mathrm{O}}=\underline{\underline{H}}^{\text {ref }}\left(n_{x_{1}}, n_{x_{2}}+\frac{1}{2}, n_{x_{3}}-\frac{1}{2}\right), \\
& \underline{\underline{H}}_{\text {ring }}^{\mathrm{O}-\mathrm{e}}=\underline{\underline{H}}^{\text {ref }}\left(n_{x_{1}}+\frac{1}{2}, n_{x_{2}}, n_{x_{3}}-\frac{1}{2}\right), \\
& \underline{\underline{H}}_{\text {ring }}^{\mathrm{O}-\mathrm{O}}=\underline{\underline{H}}^{\text {ref }}\left(n_{x_{1}}+\frac{1}{2}, n_{x_{2}}-\frac{1}{2}, n_{x_{3}}\right) .
\end{aligned}
$$

To account for the presence of quasiparticles, we treat $\hat{n}_{1}^{\prime}$ and $\hat{n}_{2}^{\prime}$ of the Hamiltonian $\hat{H}_{\text {ring }}$ in Eq. (4) as single-electronnumber operators, instead of Cooper-pair number, and the operators $\cos \left(\hat{\phi}_{1}^{\prime}\right), \cos \left(\hat{\phi}_{2}^{\prime}\right)$, and $\cos \left(\hat{\phi}_{1}^{\prime}+\hat{\phi}_{2}^{\prime}\right)$ now describe tunneling of two-electron charges [52]. In the single-electron basis $\left\{\left|n_{1}^{\prime}, n_{2}^{\prime} ; n_{0}\right\rangle ; n_{1}^{\prime}, n_{2}^{\prime} \in \mathbb{Z}\right\}$, ordered to group states within each charge sector, the ring Hamiltonian $\hat{H}_{\text {ring }}^{\prime}$ is expressed as

\footnotetext{
${ }^{5}$ Note from Eq. (2) that $\hat{n}_{1}^{\prime}$ and $\hat{n}_{2}^{\prime}$ represent charge parities of islands 1 and 2 .
} 
a diagonal block matrix $H_{\text {ring }}^{\prime}$ with four blocks corresponding to the Hamiltonians of the four sectors,

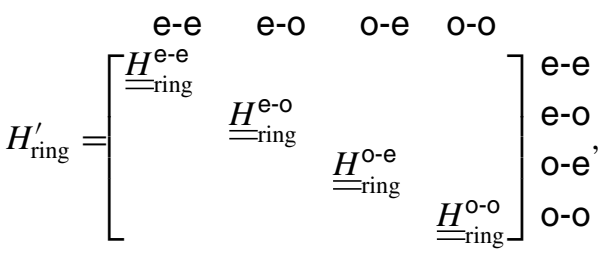

where $\underline{\underline{H}}_{\text {ing }}^{\mathrm{e}-\mathrm{e}}$ is a matrix representation of $\hat{H}_{\text {ring }}^{\mathrm{e}-\mathrm{e}}$ with $n_{1}^{\prime}$ and $n_{2}^{\prime}$ both being even valued and analogously for the other elements. Blank entries in Eq. (36) are taken to be zero.

The block structure of $\hat{H}_{\text {ring }}^{\prime}$ stems from the fact the operators $\hat{n}_{1}^{\prime}, \hat{n}_{2}^{\prime}, \cos \left(\hat{\phi}_{1}^{\prime}\right), \cos \left(\hat{\phi}_{2}^{\prime}\right)$, and $\cos \left(\hat{\phi}_{1}^{\prime}+\hat{\phi}_{2}^{\prime}\right)$ respect charge parities of the ring islands, so that the ring Hamiltonian does not couple the quasiparticle sectors.

The quasiparticle tunneling operator $\hat{T}_{23}=\sin \left(\hat{\phi}_{2}^{\prime} / 2\right)$ couples between sector pairs (e-e, e-o) and (o-e, o-o) [as depicted in Fig. 6(b)], so in sector blocks it takes the form

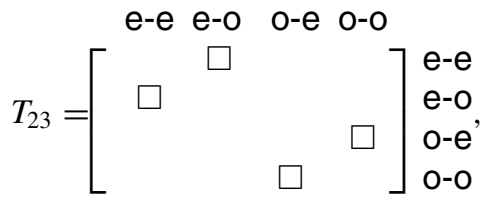

where $\square$ indicates a nonzero block submatrix. Similar block forms for other tunneling operators $\hat{T}_{12}=\sin \left(\left(\hat{\phi}_{1}^{\prime}+\hat{\phi}_{2}^{\prime}\right) / 2\right)$ and $\hat{T}_{31}=\sin \left(\hat{\phi}_{1}^{\prime} / 2\right)$ are

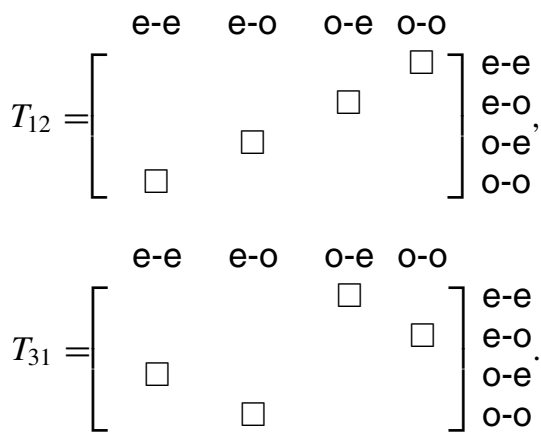

\section{B. Fluctuations between charge-parity sectors}

Having identified the four charge-parity sectors, here we evaluate the transition rates between them and compute their respective circulation. To this end, we derive the master equation for the ring density operator $\rho^{\prime}$ in the presence of quasiparticle tunneling (see Appendix F for derivation)

$$
\begin{aligned}
\dot{\rho}^{\prime}(t)= & -i\left[\hat{H}_{\text {ring }}^{\prime}-i \sqrt{\Gamma} \sum_{j}\left(\beta_{j} e^{-i \omega_{d} t} \hat{q}_{j,+}-\text { H.c. }\right), \rho^{\prime}(t)\right] \\
& +\sum_{j} \sum_{s} \sum_{k>k^{\prime}} \Gamma_{k, s ; k^{\prime}, s}^{(j)} \mathcal{D}\left[\left|k^{\prime}, s\right\rangle\langle k, s|\right] \rho^{\prime}(t) \\
& +\sum_{j \neq j^{\prime}} \sum_{s, s^{\prime}} \sum_{k, k^{\prime}} \Gamma_{k, s ; k^{\prime}, s^{\prime}}^{\left(j j^{\prime}\right)} \mathcal{D}\left[\left|k^{\prime}, s^{\prime}\right\rangle\langle k, s|\right] \rho^{\prime}(t),
\end{aligned}
$$

where $j$ and $j^{\prime}$ index the islands, $s$ and $s^{\prime}$ label the quasiparticle sectors $\{\mathrm{e}-\mathrm{e}, \mathrm{e}-\mathrm{O}, \mathrm{O}-\mathrm{e}, \mathrm{O}-\mathrm{O}\}, k$ and $k^{\prime}$ index the ring eigenstates, and $|k, s\rangle$ denotes a ring eigenstate $|k\rangle$ in the sector $s$. In Eq. (40) the second line describes the inner-sector relaxation transition due to couplings to the waveguides with the rate $\Gamma_{k, s ; k^{\prime}, s}^{(j)}=\Gamma\left|\left\langle k^{\prime}, s\left|\hat{q}_{j}\right| k, s\right\rangle\right|^{2}$, whereas the third line describes the intersector jump (i.e., quasiparticle tunneling) from a state $|k, s\rangle$ in the sector $s$ to another state $\left|k^{\prime}, s^{\prime}\right\rangle$ in the sector $s^{\prime}$ with the rate $\Gamma_{k, s ; k^{\prime}, s^{\prime}}^{\left(j j^{\prime}\right)}$ given by [53]

$$
\Gamma_{k, s ; k^{\prime}, s^{\prime}}^{\left(j j^{\prime}\right)}=\left|\left\langle k^{\prime}, s^{\prime}\left|\hat{T}_{j j^{\prime}}\right| k, s\right\rangle\right|^{2} S_{\mathrm{qp}}\left(\omega_{k, s ; k^{\prime}, s^{\prime}}\right),
$$

where the sector-coupling operator $\hat{T}_{j j^{\prime}}$ is given explicitly in Fig. 6(b) for each intersector transition, $\omega_{k, s ; k^{\prime}, s^{\prime}}$ is the transition energy between the states $|k, s\rangle$ and $\left|k^{\prime}, s^{\prime}\right\rangle$, and $S_{\mathrm{qp}}(\omega)$ is the quasiparticle spectral density. For a relaxation process with $\omega>0, S_{\mathrm{qp}}(\omega)$ is given by $[50,53]$

$$
\begin{aligned}
S_{\mathrm{qp}}(\omega)= & \frac{16 E_{J}}{\pi} \int_{0}^{\infty} d x \frac{1}{\sqrt{x} \sqrt{x+\omega / \Delta}} \\
& \times(f[(1+x) \Delta]\{1-f[(1+x) \Delta+\omega]\}),
\end{aligned}
$$

where $f[E]$ is the quasiparticle distribution function. At equilibrium, one would expect that $f[E]$ is of the form $1 /\left(\exp \left(E / k_{B} T\right)+1\right)[54]$, but nonequilibrium quasiparticles may be present modifying $f[E]$ [52]. For an excitation process with $\omega<0$, in Eq. (42) we make replacements $x \rightarrow x-\omega / \Delta$ and $\omega \rightarrow-\omega$.

At equilibrium and in the limit of high frequency, $\delta E \ll$ $\omega \ll \Delta$ with $\delta E$ the characteristic energy of quasiparticles [50,53], we can approximate $S_{\mathrm{qp}}(\omega)=\left(8 E_{J} / \pi\right) \sqrt{2 \Delta / \omega} x_{\mathrm{qp}}$, where $x_{\mathrm{qp}}$ is the quasiparticle density normalized by the Cooper-pair density:

$$
x_{\mathrm{qp}}=\sqrt{2 \pi k_{B} T / \Delta} e^{-\Delta / k_{B} T} .
$$

At $T \approx 20 \mathrm{mK}$ and for aluminum superconductors with $\Delta \approx 1.76 k_{B} T_{c}$ and $T_{c} \approx 1.35 \mathrm{~K}$ [52], $x_{\mathrm{qp}}$ should be of order $10^{-53}$, effectively suppressing quasiparticle tunneling in equilibrium BCS superconductors. Experimentally observed results for superconducting circuits nonetheless showed that $x_{\mathrm{qp}} \approx 10^{-8}-10^{-6}$ [55-57]. This indicates a small but nonnegligible population of nonequilibrium quasiparticles. The origin of these is not certain [58], but may arise from stray photons $[48,59]$, ionizing radiation from surrounding radioactive materials [60,61], and cosmic rays [55]. Further, electrons and photon baths can be out of equilibrium, so that electrons are typically hotter than the base fridge temperature [62]. In any case, we implicitly assume an empirical value for $x_{\mathrm{qp}}$.

To include nonequilibrium quasiparticles, in Eq. (43) we replace the base temperature $T(\approx 20 \mathrm{mK})$ with an effective quasiparticle one $T_{\mathrm{qp}} \approx 200 \mathrm{mK}$ [48,52]. For $E_{J} \sim$ $2 \pi \times 10 \mathrm{GHz}$ and $E_{C_{\Sigma}} / E_{J}=0.35$ we numerically find $\left|\left\langle k^{\prime}, s^{\prime}\left|\hat{T}_{j j^{\prime}}\right| k, s\right\rangle\right|^{2} \sim 10^{-2}-10^{-1}$ and $\omega_{k, s ; k^{\prime}, s^{\prime}} \sim 2 \pi \times 10 \mathrm{GHz}$, so that for $\Delta \approx 1.76 k_{B} T_{c}$ with $T_{c} \approx 1.35 \mathrm{~K}$ the quasiparticleinduced transition rate $\Gamma_{k, s ; k^{\prime}, s^{\prime}}^{\left(j j^{\prime}\right)}$ will be of order $0.1-1 \mathrm{kHz}$. This corresponds to quasiparticle lifetime of order $0.1-1 \mathrm{~ms}$ as observed in several experiments $[25,49,63,64]$. The quasiparticle temperature $T_{\mathrm{qp}}$ determines the quasiparticle-tunneling rate (i.e., the sector fluctuation rate) and does not affect the circulation performance of each quasiparticle sector. In the 
absence of quasiparticle fluctuations, the parameters would be chosen to optimize the fidelity in the fixed quasiparticle sector. Quasiparticle-trapping techniques such as normal metal traps $[24,25,30]$ and gap engineering [31-33] can be used to suppress nonequilibrium quasiparticle population. This effectively reduces the quasiparticle temperature $T_{\mathrm{qp}}$ and subsequently the quasiparticle-tunneling rate. A long period free of quasiparticle-tunneling events in the circulator will benefit device calibration and make the circulation performance stable.

\section{Symmetric Josephson-junction ring}

Since its Hamiltonian is block diagonal across the chargeparity sectors, the circulator ring will evolve within one particular sector, with intermittent, incoherent transitions between the sectors when quasiparticle tunneling events occur. Accordingly, in Appendix F we unravel the master equation in Eq. (40) into a stochastic jump evolution equation $[65,66]$ with intermittent intersector jumps. This allows us to compute the circulation performance of the device within a given quasiparticle sector during intervals in which no quasiparticle jumps occur. We note that the quasiparticle tunneling rate $\Gamma_{k, s ; k^{\prime}, s^{\prime}}^{\left(j j^{\prime}\right)}(\sim 1 \mathrm{kHz})$ is much smaller than the waveguide coupling $\Gamma(\sim 100 \mathrm{MHz})$, so $\Gamma_{k, s ; k^{\prime}, s^{\prime}}^{\left(j j^{\prime}\right)}$ has a negligible effect on the spectra within each quasiparticle sector. The principle effect of the quasiparticle-tunneling terms is just to drive transitions between sectors, with an intersector transition rate given by $\Gamma_{k, s ; k^{\prime} s^{\prime}}^{\left(j j^{\prime}\right)}$. In what follows we compare the circulation performance in each quasiparticle sector, considering both ideal symmetric rings, and realistic asymmetric rings in which the junctions are not identical.

In Fig. 7 we show variation of the scattering-matrix fidelity $F\left(S_{\text {sym }}, S_{\text {ideal }}\right)$ as a function of the reduced external flux $\phi_{x}$ and the driving frequency $\omega_{d}$ for a symmetric circulator ring with symmetric charge biases $\left(n_{x_{j}}=1 / 3\right.$ for $j=$ $1,2,3)$ in the four quasiparticle sectors. We note that here for illustration purposes we increase the coupling strength $\Gamma$ defined in Eq. (11) by effectively choosing a higher waveguide impedance $Z_{\mathrm{wg}}=200 \Omega$; smaller $Z_{\mathrm{wg}}$ reduces the bandwidth proportionally.

For the sector e-e in Fig. 7(a) we observe that a highfidelity region (dark red) and a low-fidelity region (blue) are symmetric about $\phi_{x}=\pi$. Such symmetry is owing to the mirror symmetry of the eigenstates of the circulator ring with respect to a half-quantum-flux bias, by which the high-fidelity region yields strong clockwise signal circulation while the low-fidelity region yields counterclockwise signal circulation (see also Ref. [19]).

The optimal working point in Fig. 7(a) is found at $\left(\phi_{x}^{\diamond}, \omega_{d}^{\diamond}\right) \approx\left(2.11,0.77 E_{J}\right)$ (labeled by the $\diamond$ symbol), in which the driving frequency $\omega_{d}^{\star}$ lies in between the first two excited-state eigenenergies $\omega_{1}$ and $\omega_{2}$, as expected from the condition in Eq. (28). Around this optimal working point, the bandwidth evaluated from the inset in Fig. 7(a) is around $0.01 E_{J}$. For $E_{J} \sim 2 \pi \times 10 \mathrm{GHz}$, the bandwidth is $2 \pi \times 100 \mathrm{MHz}$. This is consistent with the estimate made from Eq. (11), which yields the waveguide coupling strength $\Gamma \sim 0.01 E_{J}$ for $C_{c} / C_{\Sigma} \approx 0.31, Z_{\mathrm{wg}}=200 \Omega, \omega_{d} \approx 0.77 E_{J}$
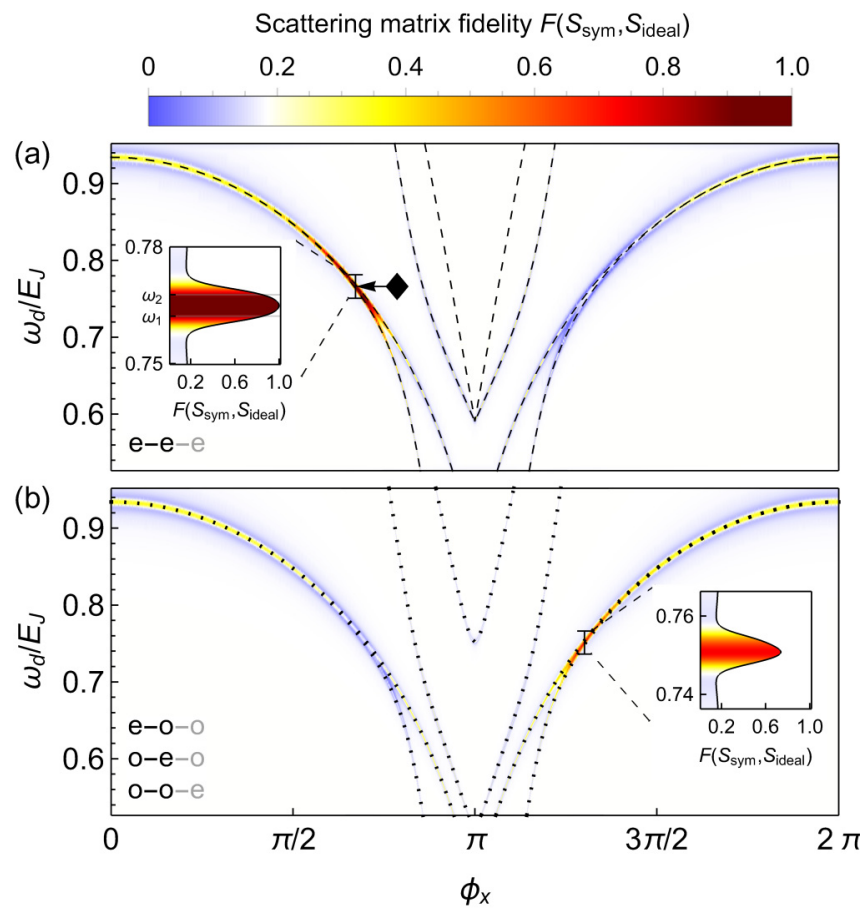

FIG. 7. Dependence on the nondimensional flux bias $\phi_{x}$ and the driving frequency $\omega_{d}$ of the scattering-matrix fidelity $F\left(S_{\text {sym }}, S_{\text {ideal }}\right)$ for a symmetric circulator ring within (a) the sector e-e(-e) and (b) the sectors $\mathrm{e}-\mathrm{o}(-\mathrm{o}), \mathrm{o}-\mathrm{e}(-\mathrm{o})$, and $\mathrm{o}-\mathrm{o}(-\mathrm{e})$. Here, owing to the junction and charge-bias symmetries, the scattering-matrix fidelities within the three sectors e-o, o-e, and o-o are exactly the same. The insets in both panels show the bandwidth of the circulator at a fixed value of $\phi_{x}$ that yields a highest fidelity. The dashed lines in (a) indicate the transition energies between the ground state of the ring and different excited states in the quasiparticle sector e-e. The $\checkmark$ symbol indicates the optimal working point. The dotted lines in (b) indicate transition energies in the sector e-o (which is the same as in o-e and o-o for a symmetric device). Relevant parameters are the same as in Fig. 3 but with $n_{x_{j}}=1 / 3(j=1,2,3)$. We also note that for illustration purposes here and in Fig. 8 we use a higher waveguide impedance $Z_{\mathrm{wg}}=200 \Omega$ to increase the coupling strength $\Gamma$.

(see Appendix D for detailed parameter values used in simulations).

We note that a background circulation fidelity at about 0.18 for a noncirculating device is indicated by white in the color scale in Fig. 7 (and Fig. 8 as well). This background fidelity is present due to the fact that when the driving frequency is far off resonant with respect to the excited-state energies, transmission of signals in the circulator is very small and there is only reflection. In particular, in Eq. (16) when $\Delta \omega_{k} / \Gamma \gg \gamma_{k},\left|\left\langle k\left|\hat{q}_{j}\right| 0\right\rangle\left\langle 0\left|\hat{q}_{i}\right| k\right\rangle\right|$ due to large detuning $\Delta \omega_{k}$, the second part of Eq. (16) is close to zero, rendering the scattering matrix very similar to an identity matrix. In this case one finds $F\left(|S|, S_{\text {ideal }}\right) \simeq F\left(\mathbb{1}, S_{\text {ideal }}\right) \approx 0.18$.

In the other quasiparticle sectors e-o, o-e, and o-o, the scattering-matrix fidelities are exactly identical for a symmetric circuit and are shown in Fig. 7(b). Comparing Figs. 7(a) and 7(b), the locations of the high-fidelity and low-fidelity regions are exchanged. Concretely, at $\left(\phi_{x}^{\star}, \omega_{d}^{\Downarrow}\right)$ in Fig. 7(a), we find $F\left(S_{\text {sym }}, S_{\text {ideal }}\right) \approx 0.99$ and $\left(S_{21}, S_{31}\right) \approx(0.001,0.996)$. 

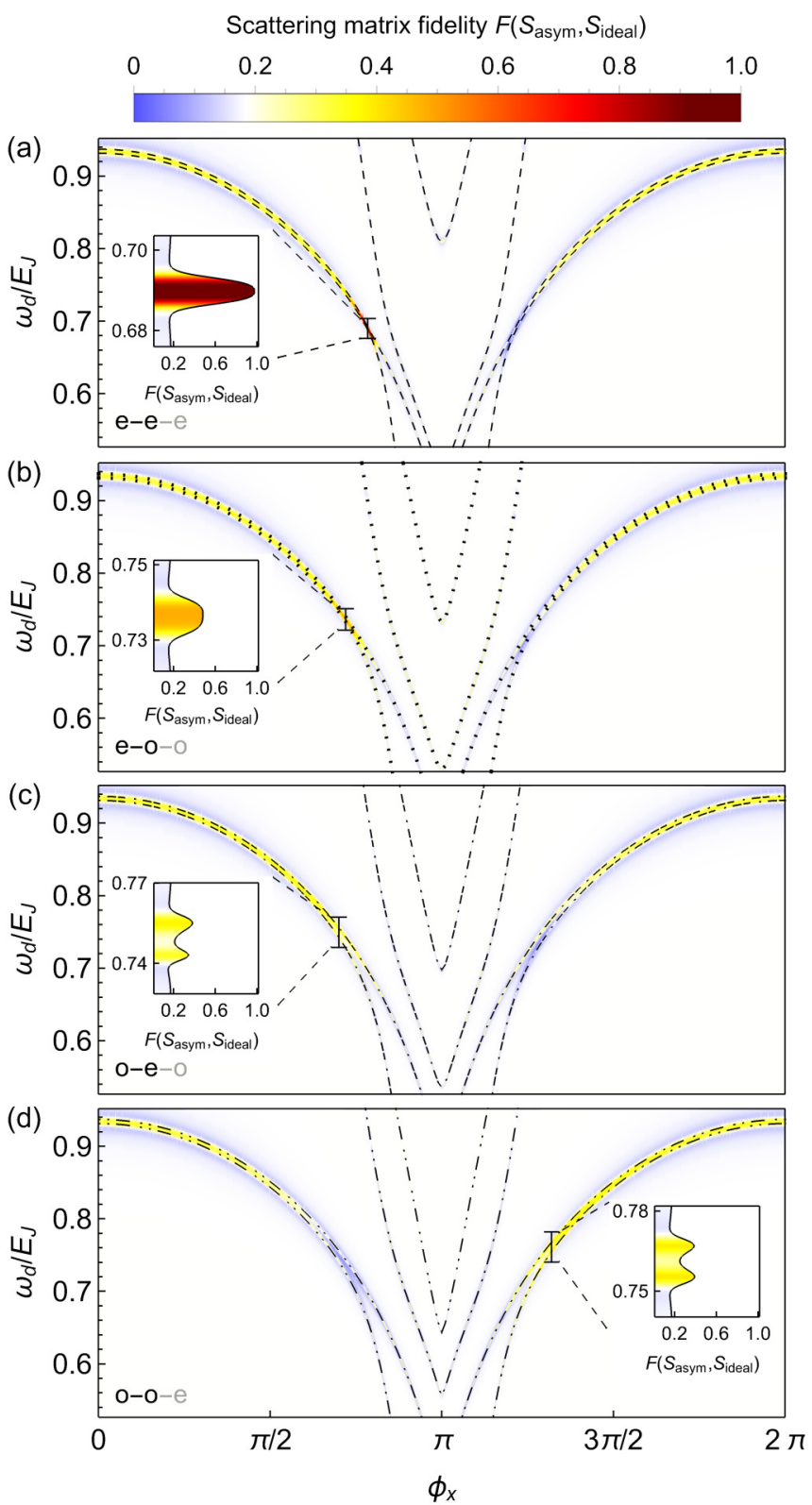

FIG. 8. Dependence on the nondimensional flux bias $\phi_{x}$ and the driving frequency $\omega_{d}$ of the fidelity of the scattering matrix $F\left(S_{\text {asym }}, S_{\text {ideal }}\right)$ for an asymmetric circulator ring within (a) the sector e-e(-e), (b) the sector e-o(-o), (c) the sector o-e(-o), and (d) the sector $\mathrm{O}-\mathrm{O}(-\mathrm{e})$. The insets in all panels show the bandwidth of the circulator at a fixed value of $\phi_{x}$ that yields a highest fidelity. Ground-to-excited-state transition energies of the circulator ring in each quasiparticle sector are also plotted (dashed, dotted, dot-dashed, and dot-dot-dashed for the four sectors, respectively). Relevant parameters are the same as in Fig. 4 but for illustration purposes we choose $Z_{\mathrm{wg}}=200 \Omega$ to increase the coupling strength, which results in $\delta E_{J} / \Gamma \sim 1$ and subsequently an optimized fidelity in (a) near 1 consistent with the analysis in Fig. 5(b).

In contrast, at the same working point in Fig. 7(b) we find $F\left(S_{\text {sym }}, S_{\text {ideal }}\right) \approx 0.14$ and $\left(S_{21}, S_{31}\right) \approx(0.622,0.274)$. These indicate significant reverse of signal circulation from clockwise to counterclockwise and the adverse influence of quasiparticle tunneling. Assuming the device is circulating signals clockwise at the high-fidelity region in the sector e-e as in Fig. 7(a), then an event of tunneling of a quasiparticle suddenly transforms the circulator to the other sectors and reverses the circulation direction as in Fig. 7(b).

\section{Asymmetric Josephson-junction ring}

We consider the same junction asymmetry as in Sec. IV B with $E_{J_{1}} / E_{J}=1, E_{J_{2}} / E_{J}=1.01$, and $E_{J_{3}} / E_{J}=$ 0.99 . We numerically optimize the scattering-matrix fidelity $F\left(S_{\text {asym }}, S_{\text {ideal }}\right)$ for the sector e-e and find its optimal value $(\approx 0.97)$ at $\left(\phi_{x}, \omega_{d}, n_{x_{1}}, n_{x_{2}}, n_{x 3}\right)=\left(2.46,0.69 E_{J}, 0.10\right.$, $0.19,0.84)$. The optimal working points for the other sectors are obtained by shifting the relevant charge biases by half of a Cooper pair while keeping the external flux and the driving frequency the same. For example, for the sector e-o we find the optimal fidelity (also $\approx 0.97)$ at $\left(\phi_{x}, \omega_{d}, n_{x_{1}}, n_{x_{2}}, n_{x 3}\right)=$ $\left(2.46,0.69 E_{J}, 0.10,0.19-1 / 2,0.84+1 / 2\right)$, and similarly for the sectors o-e and o-o. In what follows, we fix the charge bias configuration at $\left(n_{x_{1}}, n_{x_{2}}, n_{x 3}\right)=(0.10,0.19,0.84)$ to be the same for all sectors, corresponding to the experimental reality that we assume the circulator to be in the sector e-e at all times; quasiparticles will therefore degrade the performance. We plot the scattering fidelity versus $\phi_{x}$ and $\omega_{d}$ for the four sectors in Fig. 8.

Figure 8 shows that the four sectors have quite different performances. The sectors e-e, e-o, and o-e share the same high-fidelity region with $2.1 \leqslant \phi_{x} \leqslant 2.5$ but with decreasing efficiencies [see the insets in Figs. 8(a)-8(c)], while the sector o-o has its high-fidelity region mirror-flipped compared to those in the other sectors. This is different from the case of a symmetric circuit considered previously which exhibits exchange of the high-fidelity and low-fidelity regions in the sector e-e and the other sectors. Such a difference is a result of junction asymmetry $\delta E_{J}=0.01 E_{J}$ making the sectors e-o, $\mathrm{o}-\mathrm{e}$, and o-o no longer equivalent as in the symmetric-circuit case. Quasiparticle-tunneling-induced jumps between these different sectors will make the circulator operate unreliably.

\section{Composition of quasiparticle spectra}

Since circulation in our system is a resonant effect, reflection or transmission measurements of the circulator ring will reveal its energy spectrum. Each quasiparticle sector has a distinct spectrum, so measurements performed on a timescale longer than quasiparticle lifetimes will show all the spectra from the four sectors superimposed. Coexistence of the even and odd sectors has been observed in experiments with the single-Cooper-pair transistor and the Cooper-pair-box or transmon qubit $[31,32,48,49]$ that feature the "eye pattern" composing of both even and odd transitions.

In Fig. 9 we show the four sector spectra with the first four excited-state energies $\omega_{k}(k=1,2,3,4$ from bottom to top with different colors) as functions of the reduced external flux $\phi_{x}$ for an asymmetric circulator ring. We numerically compute the eigenenergies of the Hamiltonian, Eq. (36), for each quasiparticle sector (i.e., we diagonalize the closed system as for the dashed lines in Fig. 8 disregarding waveguideor quasiparticle-induced decoherence, and then compose the eigenenergies together in a single plot). The multisector spectra in Fig. 9 serve as a signature of the presence of the different 


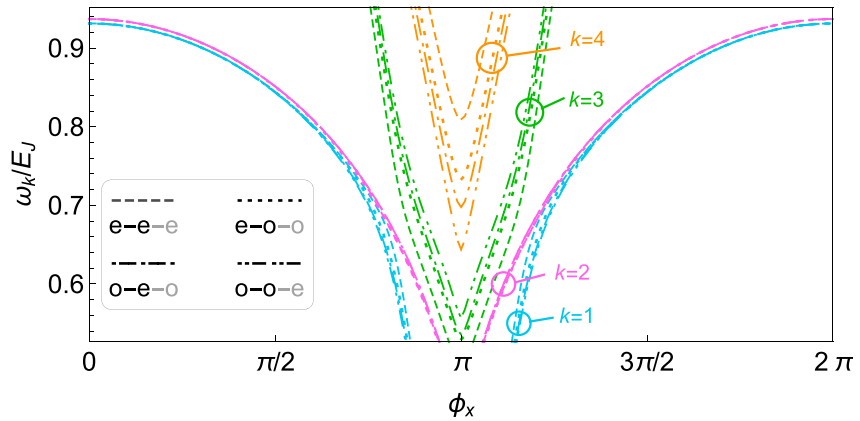

FIG. 9. Composition of the ground-to-excited-state transition energies for the first four excited states $\omega_{k}(k=1,2,3,4$ from bottom to top with different colors) from each of the four quasiparticle sectors. The line styles of the sector spectra here match those in Fig. 8. Relevant parameters are the same as in Fig. 8.

quasiparticle sectors when carrying out initial spectroscopic measurements on the circulator ring. They may also be a map to distinguish the different quasiparticle sectors.

\section{DISCUSSION}

Based on the results in the previous sections, we can sketch a three-step procedure to calibrate the Josephson-junctionring circulator as follows.

(i) First, we perform transmission or reflection measurement of the ring device to obtain its spectrum, from which we fit its energy scales (i.e., $E_{C_{\Sigma}}$ and $E_{J_{j}}$ ). We also look for any spectra overlap due to quasiparticle tunneling as in Fig. 9. No signs of such overlap indicate a device free of quasiparticles, which will facilitate finding the optimal working points.

(ii) Second, given the ring energy parameters, we numerically estimate the three charge biases, the external flux, and the driving frequency to achieve optimal circulation as done in Secs. III and IV.

(iii) Third, we incorporate a particular optimization routine into measurements of the device and optimize the scattering matrix fidelity around the parameters found in step (ii).

Of particular importance is to take into account junction asymmetry. As analyzed in Sec. IV the tolerance level of signal circulation to junction asymmetry is set by the coupling strength and in general the smaller the ratio between junction asymmetry and the coupling strength the better the scattering matrix fidelity. Superconducting quantum interference device (SQUID)-geometry junctions with tunable Josephson energies can be used to mitigate asymmetry imperfection, but might complicate device operation and introduce new noise channels. Alternatively, one can increase the coupling strength by employing high-impedance waveguides $[67,68]$ to relax constraints on device fabrication as well as enhancing the working bandwidth.

We suggest a set of fabrication parameters for the ring circulator: $E_{C_{\Sigma}} / 2 \pi \sim 3 \mathrm{GHz}, \bar{E}_{J} / 2 \pi \sim 10 \mathrm{GHz}, \Gamma / 2 \pi \sim$ $100 \mathrm{MHz}$, and $\left|\delta E_{J}\right| \lesssim 2 \Gamma$, by which $\left|\delta E_{J}\right| / \Gamma \lesssim 2$ allowing us to achieve a high circulation fidelity (see Fig. 5) with an operational bandwidth around $2 \pi \times 100 \mathrm{MHz}$. Besides, quasiparticle-trapping techniques such as normal metal traps
[24,25,30] and gap engineering [31,32] combined with careful shielding [69] can be harnessed to suppress unpaired quasiparticles, with the goal of creating a period free of quasiparticles at a timescale (about seconds or minutes $[33,58]$ ) much larger than the needed optimization time (at the order of milliseconds as estimated in Sec. IV). Conditioned on this, we expect to apply the optimization procedure presented here to a real experimental setup to calibrate the circulator device.

\section{CONCLUSION}

The passive on-chip superconducting circulator proposed in Ref. [19] is intriguing, as it operates passively and may facilitate scaling up superconducting circuit experiments. Practical operation of this device necessitates consideration of two challenging issues: tuning the various external control parameters to the optimal working points and reducing the impact of parameter instabilities. Here we have shown that even with a simple optimization routine the multiparameter optimization can be implemented quickly within less than 50 optimization steps to determine the desired operating points for the circulator device. The optimization is supported by our semianalytic treatment of the scattering problem which elucidates intuition for numerical results.

As for parameter instabilities, we have considered a detrimental type of charge noise, that is, quasiparticle tunneling. We find that tunneling of quasiparticles across the circulator junctions creates four available operating sectors, differing in the charge parities of the superconducting islands. Under the same working conditions, each sector circulates signals differently with varied circulation direction and efficiency. Stochastic jumps between the sectors due to quasiparticle tunneling subsequently may render the circulator performance inefficient. We suggest using quasiparticle-trapping and shielding techniques to reduce quasiparticle population and the quasiparticle-tunneling rate, thus potentially rendering the device unaffected by quasiparticles for a period much longer than the required optimization time.

\section{ACKNOWLEDGMENTS}

We thank Andrés Rosario Hamann for useful discussions. This research was supported by the Australian Research Council Centres of Excellence for Engineered Quantum Systems (Projects No. CE170100009 and No. CE110001013) and the Swiss National Science Foundation through NCCR Quantum Science and Technology.

\section{APPENDIX A: CIRCUIT QUANTIZATION}

\section{Combined circulator-waveguide system}

Figure 10 shows the formal representation (left) and the microscopic model (right) of the coupling between the ring island $j$ represented by the canonical flux $\Phi_{j}$ and the waveguide $j$ in which the coupling capacitance $C_{c}$ and the waveguide are treated as distributed elements. The waveguide of length $L$ is decomposed into unit cells of length $d$, so that the total unit-cell number is $N=L / d$. The waveguide capacitance and inductance per unit cell are $\mathcal{C}_{\mathrm{wg}}$ and $\ell_{\mathrm{wg}}$, yielding the waveguide capacitance and inductance per unit length as 


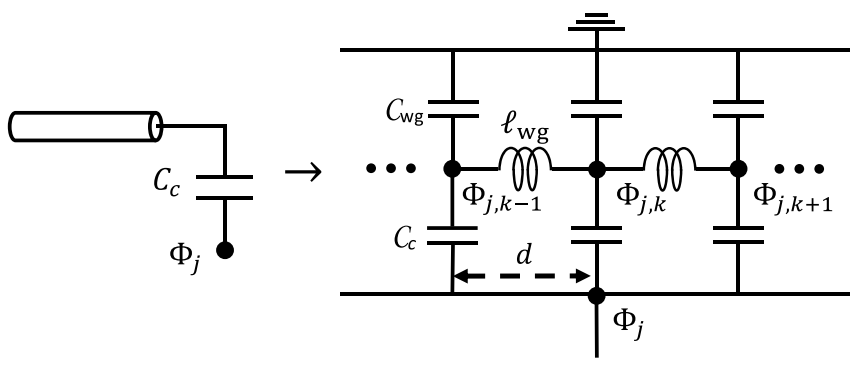

FIG. 10. Left: Formal representation of the waveguide-circulator capacitive coupling. Right: Microscopic model for this coupling in which the coupling capacitance is treated as a distributed element [35].

$\overline{\mathcal{C}}_{\mathrm{wg}}=\mathcal{C}_{\mathrm{wg}} / d$ and $\bar{\ell}_{\mathrm{wg}}=\ell_{\mathrm{wg}} / d$. The coupling capacitance $C_{c}$ has length $y$ spreading across $D=y / d$ waveguide unit cells, by which the coupling capacitance per unit cell is $\mathcal{C}_{c}=C_{c} / D$. We also assume that the waveguide length is much larger than that of the coupling capacitance, i.e., $L \gg y$.

We perform circuit quantization for the combined circulator-waveguide system assuming symmetric Josephson junctions, $E_{J j}=E_{J}$ and $C_{J j}=C_{J}$, symmetric gate capacitances, $C_{x_{j}}=C_{x}$, and symmetric coupling capacitances, $C_{c_{j}}=$ $C_{c}$. The Lagrangian of the total circuit in the absence of external biases is

$$
\begin{aligned}
\mathcal{L}_{\mathrm{tot}}= & \sum_{j=1}^{3} \frac{C_{J}}{2}\left(\dot{\Phi}_{j+1}-\dot{\Phi}_{j}\right)^{2}+\frac{C_{x}}{2} \dot{\Phi}_{j}^{2} \\
& +E_{J} \cos \left(\frac{2 \pi}{\Phi_{0}}\left(\Phi_{j+1}-\Phi_{j}\right)\right) \\
& +\sum_{j=1}^{3} \sum_{k=1}^{N} \frac{\mathcal{C}_{\mathrm{wg}}}{2} \dot{\Phi}_{j, k}^{2}-\frac{\left(\Phi_{j, k+1}-\Phi_{j, k}\right)^{2}}{2 \ell_{\mathrm{wg}}} \\
& +\sum_{j=1}^{3} \sum_{k=1}^{D} \frac{\mathcal{C}_{c}}{2}\left(\dot{\Phi}_{j, k}-\dot{\Phi}_{j}\right)^{2},
\end{aligned}
$$

where the first and second lines, respectively, represent the Lagrangians of the circulator ring and the waveguides in the absence of any coupling and the third line represents their capacitive coupling. We decompose $\mathcal{L}_{\text {tot }}$ into

$$
\mathcal{L}_{\text {tot }}=\mathcal{L}_{\text {ring }}+\mathcal{L}_{\text {wg }}+\mathcal{L}_{\text {int }},
$$

where

$$
\begin{aligned}
\mathcal{L}_{\text {ring }}= & \frac{1}{2} \dot{\boldsymbol{\Phi}} \mathbb{C} \dot{\boldsymbol{\Phi}}+E_{J} \sum_{j=1}^{3} \cos \left(\frac{2 \pi}{\Phi_{0}}\left(\Phi_{j+1}-\Phi_{j}\right)\right), \\
\mathcal{L}_{\mathrm{wg}}= & \sum_{j=1}^{3} \sum_{k=1}^{N} \frac{\mathcal{C}_{\mathrm{wg}}}{2} \dot{\Phi}_{j, k}^{2}-\frac{\left(\Phi_{j, k+1}-\Phi_{j, k}\right)^{2}}{2 \ell_{\mathrm{wg}}} \\
& +\sum_{j=1}^{3} \sum_{k=1}^{D} \frac{\mathcal{C}_{c}}{2} \dot{\Phi}_{j, k}^{2}, \\
\mathcal{L}_{\text {int }}= & -\sum_{j=1}^{3} \sum_{k=1}^{D} \mathcal{C}_{c} \dot{\Phi}_{j, k} \dot{\Phi}_{j},
\end{aligned}
$$

where $\dot{\boldsymbol{\Phi}}=\left\{\dot{\Phi}_{1}, \dot{\Phi}_{2}, \dot{\Phi}_{3}\right\}$ and

$$
\mathbb{C}=\left(\begin{array}{ccc}
C_{\Sigma}-C_{J} & -C_{J} & -C_{J} \\
-C_{J} & C_{\Sigma}-C_{J} & -C_{J} \\
-C_{J} & -C_{J} & C_{\Sigma}-C_{J}
\end{array}\right),
$$

with

$$
C_{\Sigma}=3 C_{J}+C_{x}+C_{c} .
$$

We determine the conjugate momenta $\left\{Q_{j}, Q_{j_{i} k} ; j=\right.$ $1,2,3 ; k=1, \ldots, N\}$ via the equations $Q_{j}=\partial \mathcal{L}_{\text {tot }} / \partial \Phi_{j}$ and $Q_{j, k}=\partial \mathcal{L}_{\text {tot }} / \partial \dot{\Phi}_{j, k}$ and perform Legendre transformation to compute the (classical) Hamiltonian $\mathcal{H}_{\text {tot }}$. Keeping terms to the first order of $\mathcal{C}_{c} / C_{\text {others }}$ only (that is, we assume that the unit-cell coupling capacitance $\mathcal{C}_{c}$ is much smaller than other capacitances), we find

$$
\mathcal{H}_{\text {tot }}=\mathcal{H}_{\text {ring }}+\mathcal{H}_{\mathrm{wg}}+\mathcal{H}_{\text {int }} .
$$

Concretely, $\mathcal{H}_{\text {ring }}$ is given by

$$
\mathcal{H}_{\text {ring }}=\frac{1}{2} \mathbf{Q} \mathbb{C}^{-1} \mathbf{Q}-E_{J} \sum_{j=1}^{3} \cos \left(\frac{2 \pi}{\Phi_{0}}\left(\Phi_{j+1}-\Phi_{j}\right)\right) \text {, }
$$

where $\mathbf{Q}=\left\{Q_{1}, Q_{2}, Q_{3}\right\}$. The waveguide Hamiltonian $\mathcal{H}_{\mathrm{wg}}$ is

$$
\mathcal{H}_{\mathrm{wg}}=\sum_{j=1}^{3} \sum_{k=1}^{N} \frac{Q_{j, k}^{2}}{2 \mathcal{C}_{\mathrm{wg}}}+\frac{\left(\Phi_{j, k+1}-\Phi_{j, k}\right)^{2}}{2 \ell_{\mathrm{wg}}} .
$$

Last, the interaction $\mathcal{H}_{\text {int }}$ is given by

$$
\begin{aligned}
\mathcal{H}_{\text {int }}= & \frac{\mathcal{C}_{c}}{\mathcal{C}_{\mathrm{wg}}\left(C_{x}+C_{c}\right) C_{\Sigma}} \sum_{j=1}^{3} \sum_{k=1}^{D} Q_{j, k} \\
& \times\left(\left(C_{x}+C_{c}\right) Q_{j}+C_{J} \sum_{l=1}^{3} Q_{l}\right) .
\end{aligned}
$$

We transform to the dimensionless coordinates $n=Q / 2 e$ and $\phi=2 \pi \Phi / \Phi_{0}$ and perform the first quantization to obtain the following Hamiltonians:

$$
\begin{aligned}
\hat{H}_{\text {ring }}= & \frac{(2 e)^{2}}{2} \hat{\mathbf{n}} \mathbb{C}^{-1} \hat{\mathbf{n}}-E_{J} \sum_{j=1}^{3} \cos \left(\hat{\phi}_{j+1}-\hat{\phi}_{j}\right), \\
\hat{H}_{\mathrm{wg}}= & \sum_{j=1}^{3} \sum_{k=1}^{N} E_{\mathcal{C}_{\mathrm{wg}}} \hat{n}_{j, k}^{2}+E_{\ell_{\mathrm{wg}}}\left(\hat{\phi}_{j, k+1}-\hat{\phi}_{j, k}\right)^{2}, \\
\hat{H}_{\mathrm{int}}= & \frac{(2 e)^{2} \mathcal{C}_{c}}{\mathcal{C}_{\mathrm{wg}}\left(C_{x}+C_{c}\right) C_{\Sigma}} \sum_{j=1}^{3} \sum_{k=1}^{D} \hat{n}_{j, k} \\
& \times\left(\left(C_{x}+C_{c}\right) \hat{n}_{j}+C_{J} \sum_{l=1}^{3} \hat{n}_{l}\right),
\end{aligned}
$$

where $E_{\mathcal{C}_{\mathrm{wg}}}=(2 e)^{2} /\left(2 \mathcal{C}_{\mathrm{wg}}\right)$ and $E_{\ell_{\mathrm{wg}}}=\Phi_{0}^{2} /\left(8 \pi^{2} \ell_{\mathrm{wg}}\right)$.

\section{Offset charges and external flux}

We include the offset charges and external flux to the circulator ring by simply making replacements in Eq. (A12) 
as $\hat{\mathbf{n}} \rightarrow\left(\hat{\mathbf{n}}-\mathbf{n}_{x}\right)$ with $\mathbf{n}_{x}=\left(n_{x_{1}}, n_{x_{2}}, n_{x_{3}}\right)$ and $\hat{\phi}_{j+1}-\hat{\phi}_{j} \rightarrow$ $\hat{\phi}_{j+1}-\hat{\phi}_{j}-\hat{\phi}_{x} / 3$, yielding

$$
\begin{aligned}
\hat{H}_{\text {ring }}= & \frac{(2 e)^{2}}{2}\left(\hat{\mathbf{n}}-\mathbf{n}_{x}\right) \mathbb{C}^{-1}\left(\hat{\mathbf{n}}-\mathbf{n}_{x}\right) \\
& -E_{J} \sum_{j=1}^{3} \cos \left(\hat{\phi}_{j}-\hat{\phi}_{j+1}-\frac{1}{3} \phi_{x}\right) .
\end{aligned}
$$

For the interaction Hamiltonian $\hat{H}_{\text {int }}$ in Eq. (A14), we replace $\hat{n}_{j} \rightarrow \hat{n}_{j}-n_{x_{j}}$, so that

$$
\begin{aligned}
\hat{H}_{\mathrm{int}}= & \frac{(2 e)^{2} \mathcal{C}_{c}}{\mathcal{C}_{\mathrm{wg}}\left(C_{x}+C_{c}\right) C_{\Sigma}} \sum_{j=1}^{3} \sum_{k=1}^{D} \hat{n}_{j, k} \\
& \times\left(\left(C_{x}+C_{c}\right)\left(\hat{n}_{j}-n_{x_{j}}\right)+C_{J} \sum_{l=1}^{3}\left(\hat{n}_{l}-n_{x_{l}}\right)\right) .
\end{aligned}
$$

\section{Coordinate transformation}

Since the total number of Cooper pairs on the ring is conserved, we can reduce the number of dynamical variables of the circulator system from 3 to 2 . To this end, we define new coordinates $[19,20]$

$$
\hat{\mathbf{n}}^{\prime}=A \hat{\mathbf{n}}, \quad \hat{\phi}^{\prime}=\left(A^{T}\right)^{-1} \hat{\phi},
$$

where $\hat{\mathbf{o}}=\left(\hat{o}_{1} \hat{o}_{2} \hat{o}_{3}\right)^{T}$ and

$$
A=\left(\begin{array}{ccc}
1 & 0 & 0 \\
0 & -1 & 0 \\
1 & 1 & 1
\end{array}\right)
$$

By this, $\hat{H}_{\text {ring }}$ in Eq. (A15) in terms of new coordinates is

$$
\begin{aligned}
\hat{H}_{\text {ring }}= & E_{C \Sigma}\left(\left(\hat{n}_{1}^{\prime}-\frac{1}{2}\left(n_{0}+n_{x_{1}}-n_{x_{3}}\right)\right)^{2}\right. \\
& \left.+\left(\hat{n}_{2}^{\prime}+\frac{1}{2}\left(n_{0}+n_{x_{2}}-n_{x_{3}}\right)\right)^{2}-\hat{n}_{1}^{\prime} \hat{n}_{2}^{\prime}\right) \\
& -E_{J}\left(\cos \left(\hat{\phi}_{1}^{\prime}-\frac{1}{3} \phi_{x}\right)+\cos \left(\hat{\phi}_{2}^{\prime}-\frac{1}{3} \phi_{x}\right)\right. \\
& \left.+\cos \left(\hat{\phi}_{1}^{\prime}+\hat{\phi}_{2}^{\prime}+\frac{1}{3} \phi_{x}\right)\right),
\end{aligned}
$$

where $n_{0}=\left\langle\sum_{j=1}^{3} \hat{n}_{j}\right\rangle_{\mathrm{gr}}$ is a conserved charge number evaluated from the expectation value of $\sum_{j=1}^{3} \hat{n}_{j}$ in the ground state of $\hat{H}_{\text {ring }}$ in Eq. (A12) and $E_{C \Sigma}=(2 e)^{2} / C_{\Sigma}$. The expression of $\hat{H}_{\text {int }}$ in Eq. (A16) is also altered to be

$$
\hat{H}_{\mathrm{int}}=E_{c} \sum_{k=1}^{D}\left(\hat{n}_{1, k} \hat{q}_{1}+\hat{n}_{2, k} \hat{q}_{2}+\hat{n}_{3, k} \hat{q}_{3}\right),
$$

where

$$
\begin{aligned}
\hat{q}_{1}=\hat{n}_{1}^{\prime}+n_{x_{1}}^{\prime}, \quad \hat{q}_{2}=-\hat{n}_{2}^{\prime}+n_{x_{2}}^{\prime}, \quad \hat{q}_{3}=-\hat{n}_{1}^{\prime}+\hat{n}_{2}^{\prime}+n_{x_{3}}^{\prime}, \\
n_{x_{1}}^{\prime}=c_{1}\left(n_{0}-n_{x_{2}}-n_{x_{3}}\right)-c_{2} n_{x_{1}}, \\
n_{x_{2}}^{\prime}=c_{1}\left(n_{0}-n_{x_{1}}-n_{x_{3}}\right)-c_{2} n_{x_{2}}, \\
n_{x_{3}}^{\prime}=c_{2}\left(n_{0}-n_{x_{3}}\right)-c_{1}\left(n_{x_{1}}+n_{x_{2}}\right),
\end{aligned}
$$

with $E_{c}=(2 e)^{2} \mathcal{C}_{c} /\left(\mathcal{C}_{\mathrm{wg}} C_{\Sigma}\right), c_{1}=C_{J} /\left(C_{x}+C_{c}\right)$, and $c_{2}=$ $\left(C_{J}+C_{x}+C_{c}\right) /\left(C_{x}+C_{c}\right)$. We note that the above rescaled charge biases, because of the rotating-wave approximation (used later) which considers only off-diagonal matrix elements of $\hat{q}_{j}$, are irrelevant in calculations.

\section{Waveguide normal modes and simplification of the interaction Hamiltonian}

Using the results in Ref. [35], we reexpress the waveguide Hamiltonian in Eq. (A13) in terms of its normal modes as

$$
\hat{H}_{\mathrm{wg}}=\sum_{j=1}^{3} \sum_{k=0}^{\infty} \omega_{j, k} \hat{a}_{j, k}^{\dagger} \hat{a}_{j, k},
$$

where $\omega_{j, k}=\omega_{k}=\pi k L^{-1}\left(\overline{\mathcal{C}}_{\mathrm{wg}} \bar{\ell}_{\mathrm{wg}}\right)^{-1 / 2}$ with $L, \overline{\mathcal{C}}_{\mathrm{wg}}$, and $\bar{\ell}_{\mathrm{wg}}$ respectively the length, the capacitance per unit length, and the inductance per unit length of the waveguide. The interaction $\hat{H}_{\text {int }}$ in the new waveguide modes is

$$
\hat{H}_{\text {int }}=\sum_{j=1}^{3} \sum_{k=1}^{\infty} g_{k}\left(\hat{a}_{j, k}^{\dagger}+\hat{a}_{j, k}\right) \hat{q}_{j},
$$

where $g_{k}=\left(2 C_{c} / C_{\Sigma}\right) \sqrt{2 \omega_{s} /\left(R_{K} L \overline{\mathcal{C}}_{\mathrm{wg}}\right)}$ with $R_{K}=h /(2 e)^{2} \approx$ $25.8 \mathrm{k} \Omega$ and $\hat{q}_{j}$ are given in Eq. (A21). Taking the continuum limit $[35,70]$ and expanding the lower limit of the frequencies to $-\infty[66]$ the two above Hamiltonians are recast to

$$
\begin{aligned}
& \hat{H}_{\mathrm{wg}}=\sum_{j=1}^{3} \int_{-\infty}^{\infty} d \omega \omega \hat{a}_{j}^{\dagger}(\omega) \hat{a}_{j}(\omega), \\
& \hat{H}_{\mathrm{int}}=\sum_{j=1}^{3} \int_{-\infty}^{\infty} d \omega g(\omega)\left(\hat{a}_{j}^{\dagger}(\omega)+\hat{a}_{j}(\omega)\right) \hat{q}_{j},
\end{aligned}
$$

where $g(\omega)=\left(2 C_{c} / C_{\Sigma}\right) \sqrt{2 \omega Z_{\mathrm{wg}} /\left(\pi R_{K}\right)}$ with $Z_{\mathrm{wg}}=$ $\sqrt{\bar{\ell}_{\mathrm{wg}} / \overline{\mathcal{C}}_{\mathrm{wg}}}$ the waveguide impedance. We furthermore employ the rotating-wave approximation and Markov approximation to simplify the interaction into

$$
\hat{H}_{\mathrm{int}}=\sum_{j=1}^{3} \sqrt{\frac{\Gamma}{2 \pi}} \int_{-\infty}^{\infty} d \omega\left(\hat{a}_{j}^{\dagger}(\omega) \hat{q}_{j,-}+\hat{a}_{j}(\omega) \hat{q}_{j,+}\right),
$$

where $\sqrt{\Gamma /(2 \pi)}=g\left(\omega_{d}\right)$ is the coupling strength evaluated at the driving frequency $\omega_{d}$.

\section{APPENDIX B: ADIABATIC ELIMINATION}

In this Appendix, we present the adiabatic elimination procedure presented in Ref. [28] to find a new SLH triple defined in the slow subspace of the circulator ring. We denote the initial SLH triple of the system of interest with an upper bar $(\bar{S}, \bar{L}, \bar{H})$. We define the operator

$$
\bar{K}=-\left(i \bar{H}+\frac{1}{2} \sum_{j} \bar{L}_{j}^{\dagger} \bar{L}_{j}\right) .
$$

We decompose $\bar{K}$ as

$$
\bar{K}=Y+A+B,
$$

where

$$
\begin{aligned}
& Y=P_{1} \bar{K} P_{1}, \\
& A=P_{1} \bar{K} P_{0}+P_{0} \bar{K} P_{1}, \\
& B=P_{0} \bar{K} P_{0},
\end{aligned}
$$


with $P_{0}$ the projector onto the slow subspace and $P_{1}$ onto the fast subspace. We also decompose $\bar{L}_{j}$ as

$$
\bar{L}_{j}=F_{j}+G_{j}
$$

where

$$
\begin{aligned}
F_{j} & =P_{1} \bar{L}_{j} P_{1}+P_{0} \bar{L}_{j} P_{1}, \\
G_{j} & =P_{1} \bar{L}_{j} P_{0}+P_{0} \bar{L}_{j} P_{0} .
\end{aligned}
$$

The operators (without an upper bar) in adiabatically eliminated subspace are then given by

$$
\begin{aligned}
K & =-\left(i H+\frac{1}{2} \sum_{j} L_{j}^{\dagger} L_{j}\right)=P_{0}(B-A \tilde{Y} A) P_{0}, \\
L_{j} & =\left(G_{j}-F_{j} \tilde{Y} A\right) P_{0}, \\
H & =i K+\frac{i}{2} \sum_{j} L_{j}^{\dagger} L_{j}, \\
S_{i j} & =\left(F_{i} \tilde{Y} F_{\ell}^{\dagger}+\delta_{i \ell}\right) \bar{S}_{\ell j} P_{0},
\end{aligned}
$$

where $\tilde{Y}$ satisfies

$$
Y \tilde{Y}=\tilde{Y} Y=P_{1} \text {. }
$$

For the circulator ring, its initial SLH triple is

$$
\begin{aligned}
\bar{H} & =\hat{H}_{\text {ring }}+\hat{H}_{\text {drive }}, \\
\bar{L}_{j} & =\sqrt{\Gamma} \hat{q}_{-}^{(j)}+\beta_{j} \mathbb{1}, \quad j=1,2,3, \\
\bar{S} & =\operatorname{Diag}(\mathbb{1}, \mathbb{1}, \mathbb{1}),
\end{aligned}
$$

where $\hat{H}_{\text {ring }}$ and $\hat{H}_{\text {drive }}$ are expressed in a frame rotating with the driving frequency $\omega_{d}: \hat{H}_{\text {ring }}=\sum_{k>0}\left(\omega_{k}-\omega_{d}\right)|k\rangle\langle k|$ and $\hat{H}_{\text {drive }}=-\frac{i}{2} \sqrt{\Gamma} \sum_{j=1}^{3}\left(\beta_{j} \hat{q}_{j,+}-\right.$ H.c. $)$. The ring slowsubspace and fast-subspace projectors are respectively defined as

$$
\begin{aligned}
& P_{0}=|0\rangle\langle 0|, \\
& P_{1}=\sum_{k>0}|k\rangle\langle k| .
\end{aligned}
$$

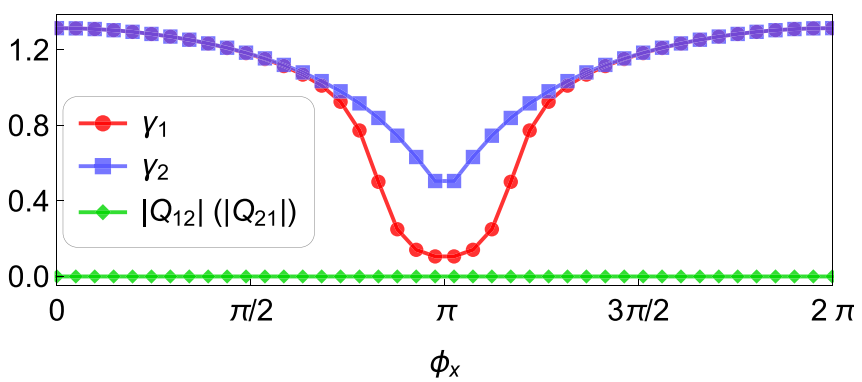

FIG. 11. $\gamma_{1}, \gamma_{2}$, and $\left|Q_{12}\right| \equiv\left|Q_{21}\right|$ as functions of the reduced external flux $\phi_{x}$, where $\gamma_{1}$ and $\gamma_{2}$ are respectively the decay rates of the excitations $|1\rangle$ and $|2\rangle$ and $Q_{k \ell}=\sum_{j=1}^{3}\left\langle 0\left|\hat{q}_{j}\right| k\right\rangle\left\langle\ell\left|\hat{q}_{j}\right| 0\right\rangle$. Note that $Q_{11} \equiv \gamma_{1}$ and $Q_{22} \equiv \gamma_{2}$. Relevant parameters are the same as in Fig. 1(b).
TABLE I. Relevant parameters used for numerical simulations in this paper.

\begin{tabular}{lc}
\hline \hline Parameter & Value \\
\hline$E_{J} / \hbar$ & $2 \pi \times 12.92 \mathrm{GHz}$ \\
$C_{J}$ & $5.76 \mathrm{fF}$ \\
$C_{x}$ & $5.95 \mathrm{fF}$ \\
$C_{c}$ & $10.60 \mathrm{fF}$ \\
$E_{C_{\Sigma}} / \hbar$ & $2 \pi \times 4.58 \mathrm{GHz}$ \\
$Z_{\mathrm{wg}}$ (Sec. IV) & $50 \Omega$ \\
$Z_{\mathrm{wg}}$ (Sec. V) & $200 \Omega$ \\
\hline \hline
\end{tabular}

Given the initial SLH triple and the projectors onto slow and fast subspaces, we carry out the computations outlined above to obtain the semianalytical expression for the scattering matrix element $S_{i j}$ as given in Eq. (16). Note that to simplify the operator $Y$, we make use of the results $\left|Q_{12}\right|,\left|Q_{21}\right| \ll$ $\left|\gamma_{1}\right|,\left|\gamma_{2}\right|$ (see next Appendix) and $\left|\beta_{j}\right|^{2} \ll \Gamma$ for weak coherent input fields.

\section{APPENDIX C: DECAY RATE COMPARISON}

In Fig. 11 we compare $\gamma_{1}, \gamma_{2}$, and $\left|Q_{12}\right| \equiv\left|Q_{21}\right|$, where $Q_{k \ell}=\sum_{j=1}^{3}\left\langle 0\left|\hat{q}_{j}\right| k\right\rangle\left\langle\ell\left|\hat{q}_{j}\right| 0\right\rangle$ for a symmetric circulator ring with identical charge biases of $1 / 3$. We clearly see that $\left|Q_{12}\right| \simeq 0$ while $\gamma_{1}, \gamma_{2}>0$, thus justifying the approximation used to obtain the property in Eq. (18). Similar results hold for the case of an asymmetric circulator ring.

\section{APPENDIX D: SIMULATION PARAMETERS}

Table I shows the values of the parameters used to perform numerical simulations in the present paper.

In Fig. 12 we plot the nonreciprocity defined by $\left|S_{12}\right|-$ $\left|S_{21}\right|$ (solid red) and the coupling strength $\Gamma$ (dashed blue) as functions of the ratio $E_{C_{\Sigma}} / E_{J}$ for a symmetric ring circulator. It is shown that nonreciprocity disappears at approximately

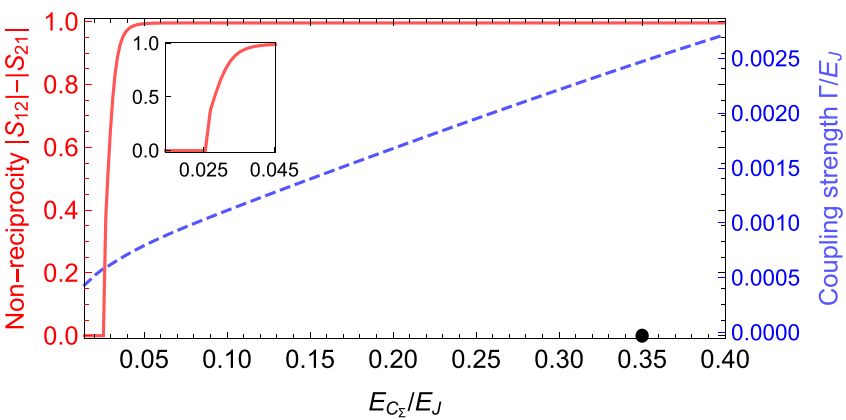

FIG. 12. Nonreciprocity represented by $\left|S_{12}\right|-\left|S_{21}\right|$ (solid red) and coupling strength $\Gamma$ (dashed blue) versus the ratio $E_{C_{\Sigma}} / E_{J}$. The inset shows the variation of $\left|S_{12}\right|-\left|S_{21}\right|$ when $E_{C_{\Sigma}} / E_{J} \leqslant 0.045$; nonreciprocity disappears near $E_{C_{\Sigma}} / E_{J}=0.025$. The coupling strength $\Gamma$ increases monotonically with $E_{C_{\Sigma}} / E_{J}$. The black dot indicates the value of $E_{C_{\Sigma}} / E_{J}(=0.35)$ used for simulations in the main text. The plot is generated for a symmetric ring. 


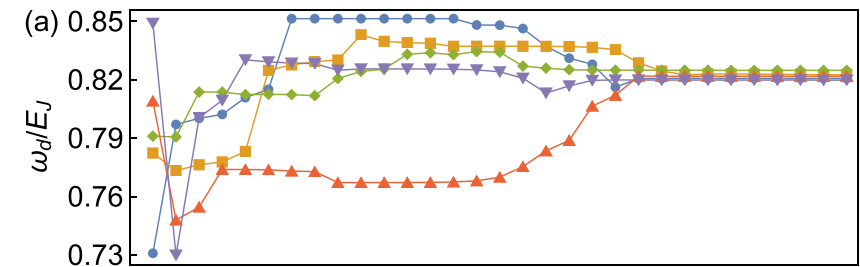

(b)

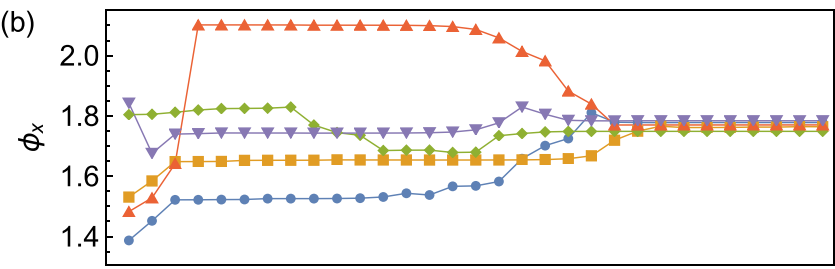

(c)

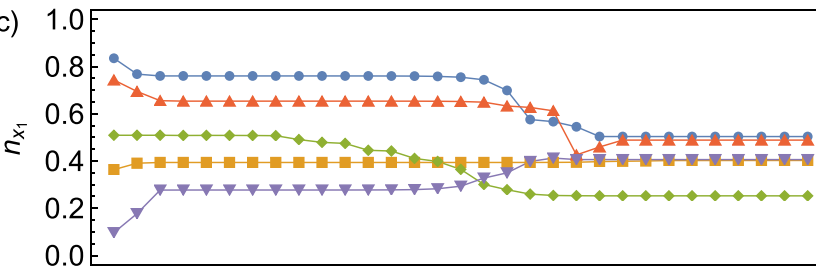

(d)

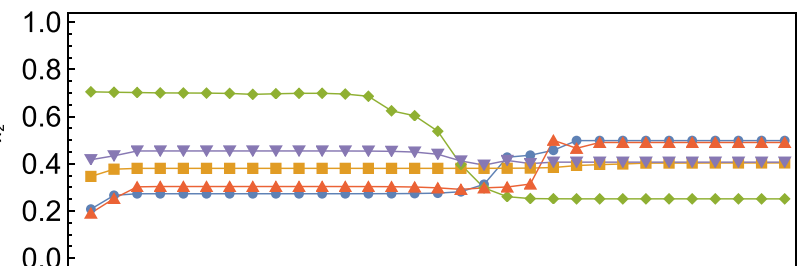

(e)

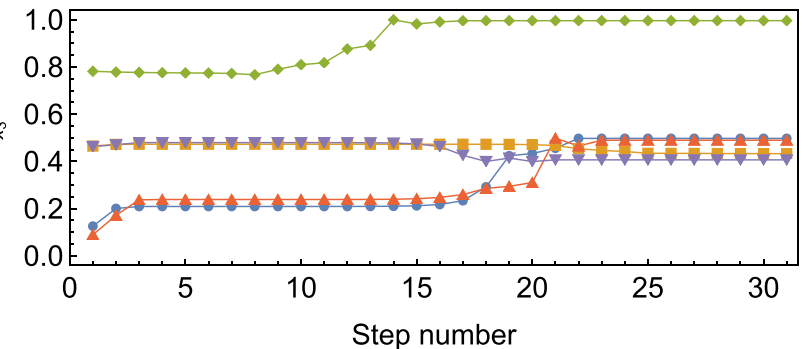

FIG. 13. Variations along the optimization in Sec. IV A for a symmetric circulator ring of (a) the driving frequency $\omega_{d}$, (b) the external flux $\phi_{x}$, and (c)-(e) the three charge biases $n_{x_{j}}$.

$E_{C_{\Sigma}} / E_{J}=0.025$ and $\Gamma$ is monotonically reduced when decreasing $E_{C_{\Sigma}} / E_{J}$.

\section{APPENDIX E: VARIATIONS OF THE WORKING PARAMETERS ALONG OPTIMIZATION}

In Fig. 13 we record the variations with respect to optimization steps of the driving frequency $\omega_{d}$ [Fig. 13(a)], the external flux $\phi_{x}$ [Fig. 13(b)], and the three charge biases $n_{x_{j}}$ [Figs. 13(c)-13(e)] for a symmetric circulator ring after the optimization in Sec. IV A. Meanwhile, in Figs. 14(a)-14(f) we show the variations of the magnitudes of the coupling matrix elements $\left|\left\langle 0\left|\hat{q}_{j}\right| k\right\rangle\right|$ for $j=1,2,3$ and $k=1,2$ to inspect the condition in Eq. (20), in Fig. 14(g) the ratio $2 \omega_{d} /\left(\omega_{1}+\omega_{2}\right)$ to inspect the condition in Eq. (28), and in Fig. 14(h) the ratio $\gamma \Gamma /\left(\omega_{2}-\omega_{1}\right)$ to inspect the condition in Eq. (29).

Figures 15 and 16 show the results for an asymmetric circulator ring.

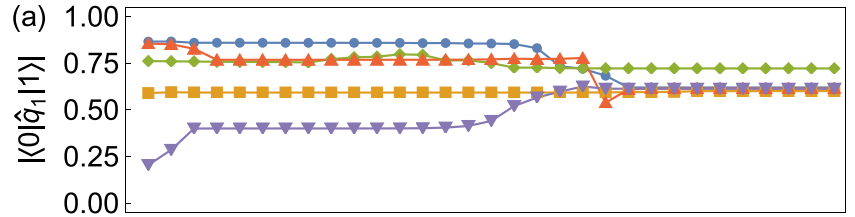

(b) 1.00

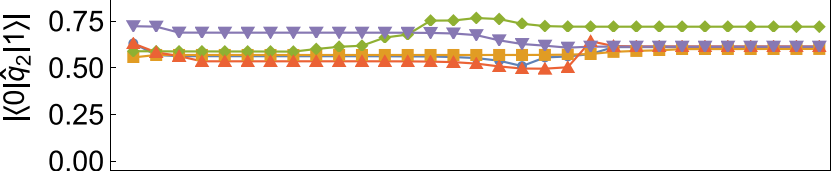

(c) 1.00

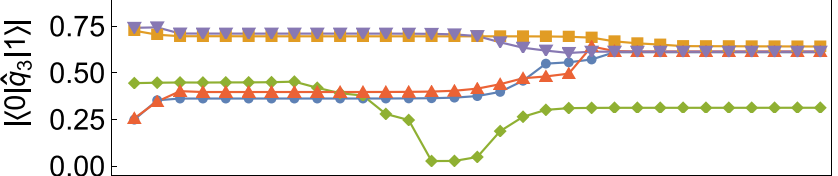

(d) 1.00

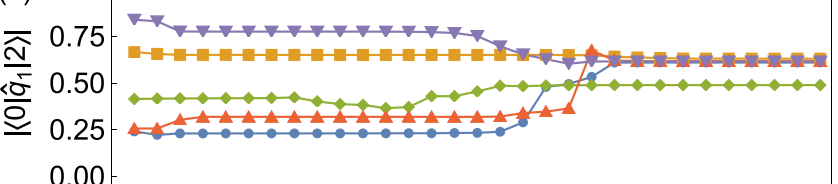

(e) 1.00

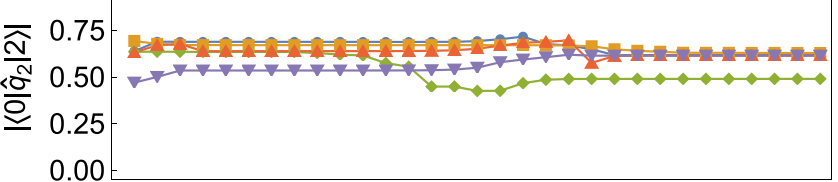

(f) 1.00

$\widehat{\widehat{A}} 0.75$

$\frac{\underline{\underline{m}}}{\underline{e}}$

0.25

0.00

(g) 1.05

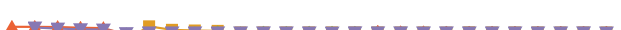

$\stackrel{+}{+} 0.95$

0.90

ก 0.85

(h)

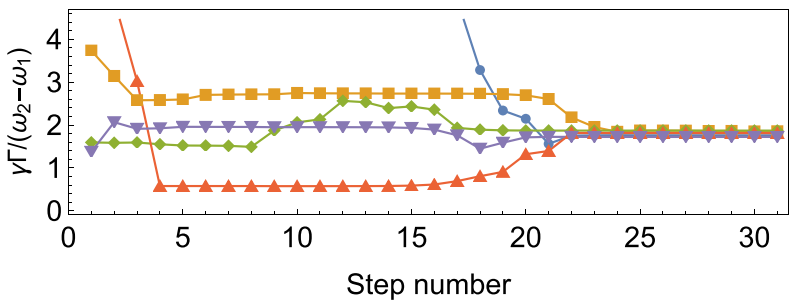

FIG. 14. Variations along the optimization in Sec. IV A for a symmetric circulator ring of $(\mathrm{a}-\mathrm{f})$ the magnitudes of the coupling matrix elements $\left|\left\langle 0\left|\hat{q}_{j}\right| k\right\rangle\right|$ for $j=1,2,3$ and $k=1,2$ to examine the condition in Eq. (20), (g) the ratio $2 \omega_{d} /\left(\omega_{1}+\omega_{2}\right)$ to examine the condition in Eq. (28), and (h) the ratio $\gamma \Gamma /\left(\omega_{2}-\omega_{1}\right)$ to examine the condition in Eq. (29).

\section{APPENDIX F: DERIVATION OF THE MASTER EQUATION IN THE PRESENCE OF QUASIPARTICLE TUNNELING}

Here we derive the master equation for the ring density operator in the presence of both coupling to waveguides and 


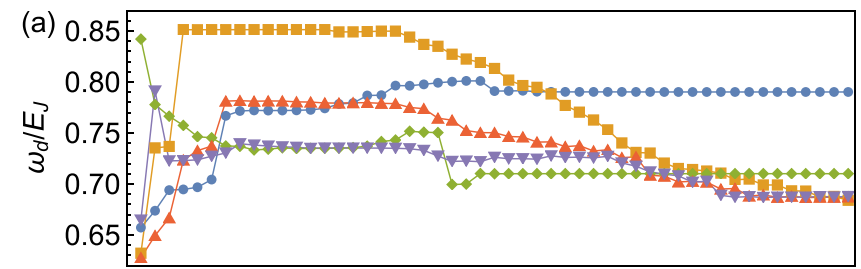

(b)

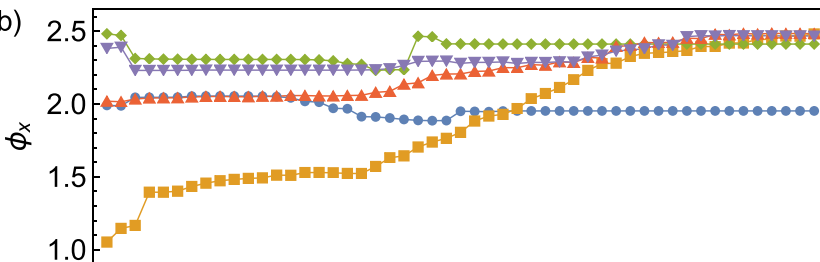

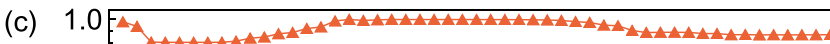

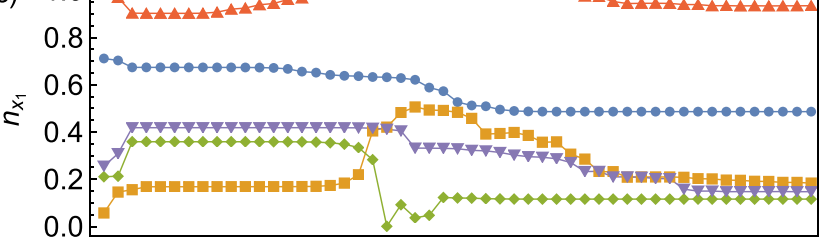

(d)

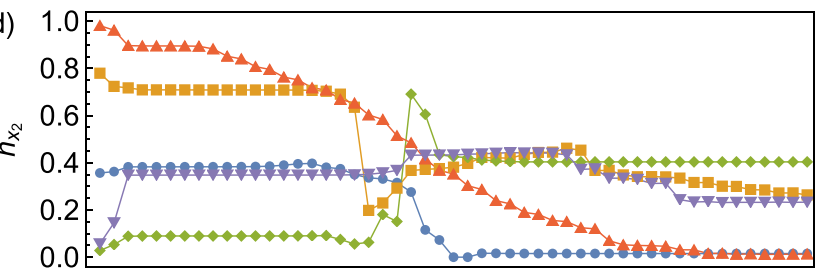

(e) 1.0 E

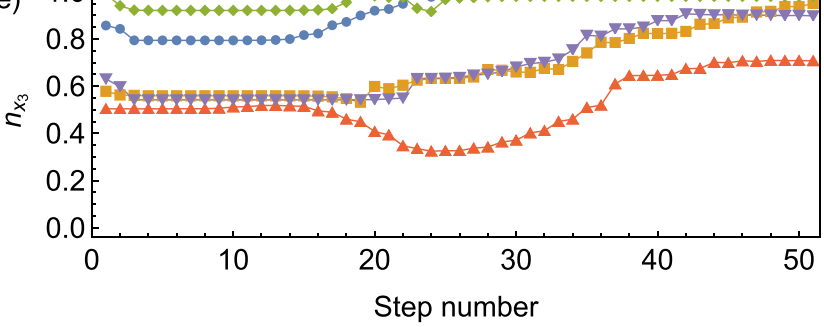

FIG. 15. Similar to Fig. 13 but for an asymmetric circulator ring and the optimization in Sec. IV B.

coupling to quasiparticles as in Eq. (40). The total waveguidering-quasiparticle Hamiltonian is [71]

$$
\hat{H}_{\text {tot }}^{\prime}=\hat{H}_{\text {ring }}^{\prime}+\hat{H}_{\mathrm{wg}}+\hat{H}_{\mathrm{int}}+\hat{H}_{\mathrm{qp}}+\hat{H}_{T},
$$

where $\hat{H}_{\text {ring }}^{\prime}$ is diagonal in sector blocks as in Eq. (36), and $\hat{H}_{\mathrm{wg}}$ and $\hat{H}_{\text {int }}$ are respectively given in Eqs. (A25) and (A26). $\hat{H}_{\mathrm{qp}}$ is the Hamiltonian of quasiparticles in the three ring islands [50]:

$$
\hat{H}_{\mathrm{qp}}=\sum_{j=1}^{3} \hat{H}_{\mathrm{qp}}^{(j)}, \quad \hat{H}_{\mathrm{qp}}^{(j)}=\sum_{n, \sigma} \epsilon_{n}^{(j)} \hat{\alpha}_{n \sigma}^{(j) \dagger} \hat{\alpha}_{n \sigma}^{(j)},
$$

where $j$ indexes the islands, $\sigma=\uparrow, \downarrow$ denotes electron spins, $\epsilon_{n}^{(j)}=\sqrt{\left(\xi_{n}^{(j)}\right)^{2}+\left(\Delta^{(j)}\right)^{2}}$ is the quasiparticle energy with $\xi_{n}^{(j)}$ the single-particle energy at level $n$ in the normal state of the island $j$ and $\Delta^{(j)}$ the gap parameter of that island, and $\hat{\alpha}_{n \sigma}^{(j)}\left(\hat{\alpha}_{n \sigma}^{(j) \dagger}\right)$ is the quasiparticle annihilation (creation) operator. $\hat{H}_{T}$ describes quasiparticle tunneling between the ring islands

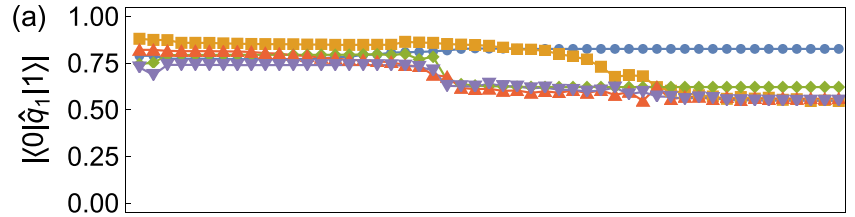

(b) 1.00
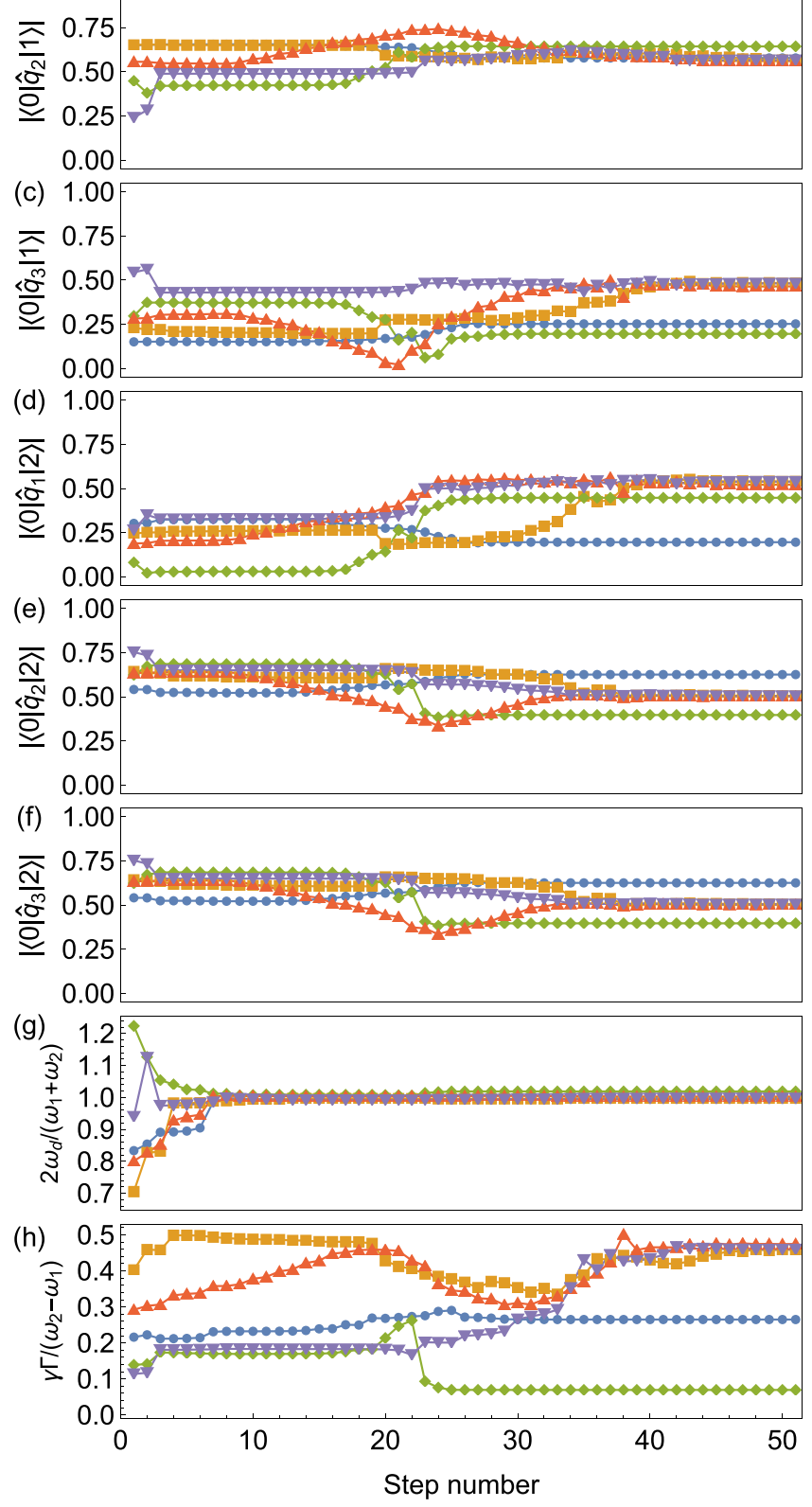

FIG. 16. Similar to Fig. 14 but for an asymmetric circulator ring and the optimization in Subsec. IV B.

[50,53]:

$$
\begin{aligned}
\hat{H}_{\mathrm{T}}= & \sum_{j \neq j^{\prime}} t_{j j^{\prime}} \sum_{n, m, \sigma} \hat{\alpha}_{n \sigma}^{(j) \dagger} \hat{\alpha}_{m \sigma}^{\left(j^{\prime}\right)}\left(e^{\frac{i}{2}\left(\hat{\varphi}_{j}-\hat{\varphi}_{j^{\prime}}\right)} u_{n}^{(j)} u_{m}^{\left(j^{\prime}\right)}\right. \\
& \left.-e^{-\frac{i}{2}\left(\hat{\varphi}_{j}-\hat{\varphi}_{j^{\prime}}\right)} v_{m}^{\left(j^{\prime}\right)} v_{n}^{(j)}\right)+ \text { H.c. },
\end{aligned}
$$

where $t_{j j^{\prime}} \ll 1$ is the tunneling amplitude between the islands $j$ and $j^{\prime}$ and is determined via the junction conductance $g_{j j^{\prime}}=4 \pi e^{2} v^{(j)} v^{\left(j^{\prime}\right)} t_{j k}^{2} / \hbar$ with $v^{(j)}$ the density of states per 
spin direction in the island $j, \hat{\varphi}_{j}$ is the phase of the island $j$, and $u_{n}^{(j)}$ and $v_{n}^{(j)}$ are Bogoliubov amplitudes and are assumed to be real. In the low-energy limit [50], we approximate $u_{m}^{(j)} \simeq v_{n}^{(j)} \simeq 1 / \sqrt{2}$, so that

$$
\hat{H}_{\mathrm{T}}=\sum_{j \neq j^{\prime}} t_{j j^{\prime}} \sum_{n, m, \sigma} \hat{\alpha}_{n \sigma}^{(j) \dagger} \hat{\alpha}_{m \sigma}^{\left(j^{\prime}\right)} \hat{T}_{j j^{\prime}}+\text { H.c. },
$$

where $\hat{T}_{j j^{\prime}}=\sin \left(\left(\hat{\varphi}_{j}-\hat{\varphi}_{j^{\prime}}\right) / 2\right)$ are the tunneling operators. In terms of the new coordinates defined in Eq. (A17), these operators are

$$
\hat{T}_{12}=\sin \left(\frac{\hat{\phi}_{1}^{\prime}+\hat{\phi}_{2}^{\prime}}{2}\right), \quad \hat{T}_{23}=\sin \left(\frac{\hat{\phi}_{2}^{\prime}}{2}\right), \quad \hat{T}_{31}=\sin \left(\frac{\hat{\phi}_{1}^{\prime}}{2}\right) .
$$

We assume that the waveguides (the bosonic baths) are in vacuum [71] and consider the effect of weak coherent driving fields later. We also assume that quasiparticles in the ring islands are near equilibrium, so that

$$
\left\langle\hat{\alpha}_{n \sigma}^{(j) \dagger}\right\rangle_{\mathrm{qp}}=\left\langle\hat{\alpha}_{n \sigma}^{(j)}\right\rangle_{\mathrm{qp}}=0, \quad\left\langle\hat{\alpha}_{n \sigma}^{(j) \dagger} \hat{\alpha}_{n \sigma}^{\left(j^{\prime}\right)}\right\rangle_{\mathrm{qp}}=\delta_{j j^{\prime}} f^{(j)}\left[\epsilon_{n}^{(j)}\right],
$$

where $f^{(j)}\left[\epsilon_{n}^{(j)}\right]$ is the distribution function of quasiparticles in the island $j$ which is assumed to be independent of spin. We decompose $\hat{H}_{\text {tot }}^{\prime}$ in Eq. (F1) into

$$
\hat{H}_{\text {tot }}^{\prime}=\hat{H}_{0}+\hat{V} \text {, }
$$

where $\hat{H}_{0}=\hat{H}_{\text {ring }}^{\prime}+\hat{H}_{\mathrm{wg}}+\hat{H}_{\mathrm{qp}}$ is the total unperturbed Hamiltonian and $\hat{V}=\hat{H}_{\text {int }}+\hat{H}_{T}$ is a perturbation to $\hat{H}_{0}$. Let $\varrho$ be the density operator of the total waveguide-ringquasiparticle system and $\rho^{\prime}=\operatorname{Tr}_{\mathrm{wg}, \mathrm{qp}}(\varrho)$ be the ring density operator. In the interaction picture, the equation of motion of $\rho^{\prime}$ is $[71]$

$$
\dot{\rho}_{I}^{\prime}(t)=-\int_{-\infty}^{t} d t^{\prime} \operatorname{Tr}_{\mathrm{wg}, \mathrm{qp}}\left\{\left[\hat{V}_{I}(t),\left[\hat{V}_{I}\left(t^{\prime}\right), \varrho_{I}\left(t^{\prime}\right)\right]\right]\right\},
$$

where

$$
\begin{aligned}
\hat{V}_{I}(t)= & e^{-i \hat{H}_{0} t} \hat{V} e^{i \hat{H}_{0} t} \\
= & \sum_{j=1}^{3} \sqrt{\frac{\Gamma}{2 \pi}} \int_{-\infty}^{\infty} d \omega\left(a_{j}^{\dagger}(\omega) e^{i \omega t} \hat{q}_{j}^{-}(t)+\text { H.c. }\right) \\
& +\left(\sum_{j \neq j^{\prime}} t_{j j^{\prime}} \sum_{n, m, \sigma} e^{i\left(\epsilon_{n}^{(j)}-\epsilon_{m}^{\left(j^{\prime}\right)}\right) t} \hat{\alpha}_{n \sigma}^{(j) \dagger} \hat{\alpha}_{m \sigma}^{j^{\prime}} \hat{T}_{j j^{\prime}}(t)+\text { H.c. }\right) .
\end{aligned}
$$

Here the operators $\hat{q}_{j}^{-}(t)$ and $\hat{T}_{j j^{\prime}}(t)$ are

$$
\begin{aligned}
& \hat{q}_{j}^{-}(t)=\sum_{s} \sum_{k>k^{\prime}}\left\langle k^{\prime}, s\left|\hat{q}_{j}\right| k, s\right\rangle e^{i \omega_{k, s ; k^{\prime}, s^{t}}}\left|k^{\prime}, s\right\rangle\langle k, s|, \\
& \hat{T}_{j j^{\prime}}(t)=\sum_{s, s^{\prime}} \sum_{k, k^{\prime}}\left\langle k^{\prime}, s^{\prime}\left|\hat{T}_{j j^{\prime}}\right| k, s\right\rangle e^{i \omega_{k, s ; k^{\prime}, s^{\prime}}}\left|k^{\prime}, s^{\prime}\right\rangle\langle k, s|,
\end{aligned}
$$

where $s$ and $s^{\prime}$ label the sector indices and $k$ and $k^{\prime}$ label the ring eigenstate indices within each sector.

The right-hand side of Eq. (F8), due to the double commutator, has four terms of which we consider only the term containing $\hat{V}_{I}(t) \hat{V}_{I}\left(t^{\prime}\right) \varrho_{I}\left(t^{\prime}\right)$. The other terms are evaluated similarly. We have

$$
\begin{aligned}
& -\int_{-\infty}^{t} d t^{\prime} \operatorname{Tr}_{\mathrm{wg}, \mathrm{qp}}\left\{\hat{V}_{I}(t) \hat{V}_{I}\left(t^{\prime}\right) \varrho_{I}\left(t^{\prime}\right)\right\} \\
& =-\frac{1}{2} \sum_{j=1}^{3} \sum_{s} \sum_{k>k^{\prime}} \Gamma_{k, s ; k^{\prime}, s}^{(j)}|k, s\rangle\left\langle k^{\prime}, s|| k^{\prime}, s\right\rangle\langle k, s| \rho_{I}^{\prime}(t) \\
& \quad-\frac{1}{2} \sum_{j \neq j^{\prime}} \sum_{s, s^{\prime}} \sum_{k, k^{\prime}} \Gamma_{k, s ; k^{\prime}, s^{\prime}}^{\left(j j^{\prime}\right)}|k, s\rangle\left\langle k^{\prime}, s^{\prime}|| k^{\prime}, s^{\prime}\right\rangle\langle k, s| \rho_{I}^{\prime}(t),
\end{aligned}
$$

where we have used the Born-Markov approximation and neglected fast oscillating terms, $\Gamma_{k, s ; k^{\prime}, s}^{(j)}$ is the inner-sector relaxation rate,

$$
\Gamma_{k, s ; k^{\prime}, s}^{(j)}=\Gamma\left|\left\langle k^{\prime}, s\left|\hat{q}_{j}\right| k, s\right\rangle\right|^{2},
$$

and $\Gamma_{k, s ; k^{\prime}, s^{\prime}}^{\left(j j^{\prime}\right)}$ is the intersector (i.e., quasiparticle-tunneling) rate,

$$
\Gamma_{k, s ; k^{\prime}, s^{\prime}}^{\left(j j^{\prime}\right)}=\left|\left\langle k^{\prime}, s^{\prime}\left|\hat{T}_{j j^{\prime}}\right| k, s\right\rangle\right|^{2} S_{\mathrm{qp}}\left(\omega_{k, s ; k^{\prime}, s^{\prime}}\right),
$$

with $\omega_{k, s ; k^{\prime}, s^{\prime}}$ the transition energy between the states $|k, s\rangle$ and $\left|k^{\prime}, s^{\prime}\right\rangle$. Here $S_{\mathrm{qp}}^{\left(j j^{\prime}\right)}(\omega)$ is the quasiparticle spectral density and for $\omega>0$ is given by [50]

$$
\begin{aligned}
S_{\mathrm{qp}}^{\left(j j^{\prime}\right)}(\omega)= & \frac{16 E_{J}^{\left(j j^{\prime}\right)}}{\pi} \int_{0}^{\infty} d x \frac{1}{\sqrt{x} \sqrt{x+\omega / \Delta}} \\
& \times\left(f^{(j)}[(1+x) \Delta]\left\{1-f^{\left(j^{\prime}\right)}[(1+x) \Delta+\omega]\right\}\right),
\end{aligned}
$$

where $E_{J}^{\left(j j^{\prime}\right)}$ is the Josephson energy of the junction connecting the islands $j$ and $j^{\prime}$. For $\omega<0$, in Eq. (F15) we make replacements $x \rightarrow x-\omega / \Delta$ and $\omega \rightarrow-\omega$. We consider equal populations on the islands, so $f^{(j)}=f^{\left(j^{\prime}\right)}$ simplifying $S_{\mathrm{qp}}^{\left(j j^{\prime}\right)}$ into Eq. (42).

Using the above results, Eq. (F8) is recast to

$$
\begin{aligned}
\dot{\rho}_{I}^{\prime}(t)= & \sum_{j=1} \sum_{s} \sum_{k<k^{\prime}} \Gamma_{k, s ; k^{\prime}, s}^{(j)} \mathcal{D}\left[\left|k^{\prime}, s\right\rangle\langle k, s|\right] \rho_{I}^{\prime}(t) \\
& +\sum_{j \neq j^{\prime}} \sum_{s, s^{\prime}} \sum_{k, k^{\prime}} \Gamma_{k, s ; k^{\prime}, s^{\prime}}^{\left(j j^{\prime}\right)} \mathcal{D}\left[\left|k^{\prime}, s^{\prime}\right\rangle\langle k, s|\right] \rho_{I}^{\prime}(t) .
\end{aligned}
$$

We transfer the master equation to the Schrodinger picture and add weak coherent driving fields [36,72], yielding

$$
\begin{aligned}
\dot{\rho}^{\prime}(t)= & -i\left[\hat{H}_{\text {ring }}^{\prime}-i \sqrt{\Gamma} \sum_{j}\left(\beta_{j} e^{-i \omega_{d} t} \hat{q}_{j,+}-\text { H.c. }\right), \rho^{\prime}(t)\right] \\
& +\sum_{j} \sum_{s} \sum_{k>k^{\prime}} \Gamma_{k, s ; k^{\prime}, s}^{(j)} \mathcal{D}\left[\left|k^{\prime}, s\right\rangle\langle k, s|\right] \rho^{\prime}(t) \\
& +\sum_{j \neq j^{\prime}} \sum_{s, s^{\prime}} \sum_{k, k^{\prime}} \Gamma_{k, s ; k^{\prime}, s^{\prime}}^{\left(j j^{\prime}\right)} \mathcal{D}\left[\left|k^{\prime}, s^{\prime}\right\rangle\langle k, s|\right] \rho^{\prime}(t),
\end{aligned}
$$

which is the master equation in Eq. (40). 
We unravel Eq. (F17) into a conditional master equation $[65,66]$

$$
\begin{aligned}
\dot{\rho}_{c, 0}^{\prime}(t)= & -i\left[\hat{H}_{\mathrm{eff}}, \rho_{c, 0}^{\prime}(t)\right]+\sum_{j} \sum_{s} \sum_{k>k^{\prime}} \Gamma_{k, s ; k^{\prime}, s}^{(j)} \\
& \times \mathcal{D}\left[\left|k^{\prime}, s\right\rangle\langle k, s|\right] \rho_{c, 0}^{\prime}(t),
\end{aligned}
$$

where $\rho_{c, 0}^{\prime}(t)$ is the conditional system density operator conditioned on when no quasiparticle jumps happen; that is, it describes evolution within one quasiparticle sector in between incoherent jumps to the other sectors, and $\hat{H}_{\text {eff }}$ is the effective no-jump Hamiltonian:

$$
\begin{aligned}
\hat{H}_{\mathrm{eff}}= & \hat{H}_{\mathrm{ring}}^{\prime}-i \sqrt{\Gamma} \sum_{j}\left(\beta_{j} e^{-i \omega_{d} t} \hat{q}_{j,+}-\text { H.c. }\right) \\
& -\frac{i}{2} \sum_{j \neq j^{\prime}} \sum_{s, s^{\prime}} \sum_{k, k^{\prime}} \Gamma_{k, s ; k^{\prime}, s^{\prime}}^{\left(j j^{\prime}\right)}|k, s\rangle\langle k, s| .
\end{aligned}
$$

The non-Hermitian part of $\hat{H}_{\text {eff }}$ [the second line in Eq. (F19)] is the system self-damping [66] given in terms of the sector-mixing operator $\hat{c}_{k, s ; k^{\prime}, s^{\prime}}=\left|k^{\prime}, s^{\prime}\right\rangle\langle k, s|$ as $|k, s\rangle\langle k, s|=$ $\hat{c}_{k, s ; k^{\prime}, s^{\prime}}^{\dagger} \hat{c}_{k, s ; k^{\prime}, s^{\prime}}$. This self-damping term is negligible compared to the Hermitian part of $\hat{H}_{\text {eff }}$ [the first line in Eq. (F19)], since $\Gamma_{k, s: k^{\prime}, s^{\prime}}^{\left(j j^{\prime}\right.} \sim 1 \mathrm{kHz}$ (as estimated in Sec. VB) is much smaller than $\omega_{k, s} \sim 2 \pi \times 10 \mathrm{GHz}$ and $\Gamma \sim 2 \pi \times 100 \mathrm{MHz}$

\section{APPENDIX G: SECTOR FLUCTUATIONS FOR AN ODD TOTAL CHARGE PARITY}

In this Appendix, we consider sector fluctuations when the total charge parity of the ring islands is odd. The four quasiparticle sectors include e-e(-o), e-o(-e), o-e(-e), and o-o(-o). For a symmetric ring circuit, we find the fidelity $F\left(S_{\text {sym }}, S_{\text {ideal }}\right)$ for the sector e-e is optimized at $\left(\omega_{d}, \phi_{x}, n_{x_{1}}, n_{x_{2}}, n_{x_{3}}\right)=$ $\left(0.77 E_{J}, 2.11,1 / 3,1 / 3,5 / 6\right)$. We fix the three charge biases and plot the fidelity versus $\omega_{d}$ and $\phi_{x}$ for the four sectors. The results are almost identical to those in Fig. 7, so we do not show them here for brevity.

We repeat the above procedure for an asymmetric circuit with the same junction asymmetries as in Fig. 8 and observe that sector fluctuations are qualitatively analogous to the results in Fig. 8.
[1] X. Gu, A. F. Kockum, A. Miranowicz, Y.-xi Liu, and F. Nori, Microwave photonics with superconducting quantum circuits, Phys. Rep. 718, 1 (2017).

[2] L. Deák and T. Fülöp, Reciprocity in quantum, electromagnetic and other wave scattering, Ann. Phys. 327, 1050 (2012).

[3] B. J. Chapman, E. I. Rosenthal, J. Kerckhoff, B. A. Moores, L. R. Vale, J. A. B. Mates, G. C. Hilton, K. Lalumière, A. Blais, and K. W. Lehnert, Widely Tunable On-Chip Microwave Circulator for Superconducting Quantum Circuits, Phys. Rev. X 7, 041043 (2017).

[4] D. Pozar, Microwave Engineering (Wiley, New York, 2011).

[5] G. Viola and D. P. DiVincenzo, Hall Effect Gyrators and Circulators, Phys. Rev. X 4, 021019 (2014).

[6] A. C. Mahoney, J. I. Colless, S. J. Pauka, J. M. Hornibrook, J. D. Watson, G. C. Gardner, M. J. Manfra, A. C. Doherty, and D. J. Reilly, On-Chip Microwave Quantum Hall Circulator, Phys. Rev. X 7, 011007 (2017).

[7] A. Kamal, J. Clarke, and M. H. Devoret, Noiseless nonreciprocity in a parametric active device, Nat. Phys. 7, 311 (2011).

[8] K. M. Sliwa, M. Hatridge, A. Narla, S. Shankar, L. Frunzio, R. J. Schoelkopf, and M. H. Devoret, Reconfigurable Josephson Circulator/Directional Amplifier, Phys. Rev. X 5, 041020 (2015).

[9] F. Lecocq, L. Ranzani, G. A. Peterson, K. Cicak, R. W. Simmonds, J. D. Teufel, and J. Aumentado, Nonreciprocal Microwave Signal Processing with a Field-Programmable Josephson Amplifier, Phys. Rev. Appl. 7, 024028 (2017).

[10] C. W. Peterson, W. A. Benalcazar, M. Lin, T. L. Hughes, and G. Bahl, Strong Nonreciprocity in Modulated Resonator Chains through Synthetic Electric and Magnetic Fields, Phys. Rev. Lett. 123, 063901 (2019).

[11] K. Fang, Z. Yu, and S. Fan, Realizing effective magnetic field for photons by controlling the phase of dynamic modulation, Nat. Photonics 6, 782 (2012).
[12] A. Kamal and A. Metelmann, Minimal Models for Nonreciprocal Amplification Using Biharmonic Drives, Phys. Rev. Appl. 7, 034031 (2017).

[13] N. A. Estep, D. L. Sounas, J. Soric, and A. Alù, Magneticfree non-reciprocity and isolation based on parametrically modulated coupled-resonator loops, Nat. Phys. 10, 923 (2014).

[14] J. Kerckhoff, K. Lalumière, B. J. Chapman, A. Blais, and K. W. Lehnert, On-Chip Superconducting Microwave Circulator from Synthetic Rotation, Phys. Rev. Appl. 4, 034002 (2015).

[15] P. Roushan, C. Neill, A. Megrant, Y. Chen, R. Babbush, R. Barends, B. Campbell, Z. Chen, B. Chiaro, A. Dunsworth, A. Fowler, E. Jeffrey, J. Kelly, E. Lucero, J. Mutus, P. J. J. O’Malley, M. Neeley, C. Quintana, D. Sank, A. Vainsencher, et al., Chiral ground-state currents of interacting photons in a synthetic magnetic field, Nat. Phys. 13, 146 (2017).

[16] E. I. Rosenthal, B. J. Chapman, A. P. Higginbotham, J. Kerckhoff, and K. W. Lehnert, Breaking Lorentz Reciprocity with Frequency Conversion and Delay, Phys. Rev. Lett. 119, 147703 (2017)

[17] A. Metelmann and A. A. Clerk, Nonreciprocal Photon Transmission and Amplification via Reservoir Engineering, Phys. Rev. X 5, 021025 (2015).

[18] K. Fang, J. Luo, A. Metelmann, M. H. Matheny, F. Marquardt, A. A. Clerk, and O. Painter, Generalized non-reciprocity in an optomechanical circuit via synthetic magnetism and reservoir engineering, Nat. Phys. 13, 465 (2017).

[19] C. Müller, S. Guan, N. Vogt, J. H. Cole, and T. M. Stace, Passive On-Chip Superconducting Circulator Using a Ring of Tunnel Junctions, Phys. Rev. Lett. 120, 213602 (2018).

[20] J. Koch, A. A. Houck, K. L. Hur, and S. M. Girvin, Time-reversal-symmetry breaking in circuit-QED-based photon lattices, Phys. Rev. A 82, 043811 (2010).

[21] V. Fatemi, A. R. Akhmerov, and L. Bretheau, Weyl Josephson circuits, Phys. Rev. Res. 3, 013288 (2021). 
[22] R. Asensio-Perea, A. Parra-Rodriguez, G. Kirchmair, E. Solano, and E. Rico, Chiral states and nonreciprocal phases in a Josephson junction ring, Phys. Rev. B 103, 224525 (2021).

[23] K. A. Matveev, M. Gisselfält, L. I. Glazman, M. Jonson, and R. I. Shekhter, Parity-Induced Suppression of the Coulomb Blockade of Josephson Tunneling, Phys. Rev. Lett. 70, 2940 (1993).

[24] P. Joyez, P. Lafarge, A. Filipe, D. Esteve, and M. H. Devoret, Observation of Parity-Induced Suppression of Josephson Tunneling in the Superconducting Single Electron Transistor, Phys. Rev. Lett. 72, 2458 (1994).

[25] N. A. Court, A. J. Ferguson, R. Lutchyn, and R. G. Clark, Quantitative study of quasiparticle traps using the single-Cooper-pair transistor, Phys. Rev. B 77, 100501(R) (2008).

[26] R. Lutchyn, L. Glazman, and A. Larkin, Quasiparticle decay rate of Josephson charge qubit oscillations, Phys. Rev. B 72, 014517 (2005).

[27] R. M. Lutchyn, L. I. Glazman, and A. I. Larkin, Kinetics of the superconducting charge qubit in the presence of a quasiparticle, Phys. Rev. B 74, 064515 (2006).

[28] J. Combes, J. Kerckhoff, and M. Sarovar, The SLH framework for modeling quantum input-output networks, Adv. Phys. X 2, 784 (2017).

[29] C. Müller, J. Combes, A. R. Hamann, A. Fedorov, and T. M. Stace, Nonreciprocal atomic scattering: A saturable, quantum Yagi-Uda antenna, Phys. Rev. A 96, 053817 (2017).

[30] J. M. Martinis, Saving superconducting quantum processors from decay and correlated errors generated by gamma and cosmic rays, npj Quantum Inf. 7, 90 (2021).

[31] J. Aumentado, M. W. Keller, J. M. Martinis, and M. H. Devoret, Nonequilibrium Quasiparticles and $2 e$ Periodicity in SingleCooper-Pair Transistors, Phys. Rev. Lett. 92, 066802 (2004).

[32] L. Sun, L. DiCarlo, M. D. Reed, G. Catelani, L. S. Bishop, D. I. Schuster, B. R. Johnson, G. A. Yang, L. Frunzio, L. Glazman, M. H. Devoret, and R. J. Schoelkopf, Measurements of Quasiparticle Tunneling Dynamics in a Band-Gap-Engineered Transmon Qubit, Phys. Rev. Lett. 108, 230509 (2012).

[33] K. Kalashnikov, W. T. Hsieh, W. Zhang, W.-S. Lu, P. Kamenov, A. Di Paolo, A. Blais, M. E. Gershenson, and M. Bell, Bifluxon: Fluxon-parity-protected superconducting qubit, PRX Quantum 1, 010307 (2020).

[34] C. W. Gardiner and M. J. Collett, Input and output in damped quantum systems: Quantum stochastic differential equations and the master equation, Phys. Rev. A 31, 3761 (1985).

[35] D. T. Le, A. Grimsmo, C. Müller, and T. M. Stace, Doubly nonlinear superconducting qubit, Phys. Rev. A 100, 062321 (2019).

[36] B. Peropadre, J. Lindkvist, I.-C. Hoi, C. M. Wilson, J. J. GarciaRipoll, P. Delsing, and G. Johansson, Scattering of coherent states on a single artificial atom, New J. Phys. 15, 035009 (2013).

[37] M. Devoret, S. Girvin, and R. Schoelkopf, Circuit-QED: How strong can the coupling between a Josephson junction atom and a transmission line resonator be?, Ann. Phys. 16, 767 (2007).

[38] A. Blais, A. L. Grimsmo, S. M. Girvin, and A. Wallraff, Circuit quantum electrodynamics, Rev. Mod. Phys. 93, 025005 (2021).

[39] E. Wiegand, B. Rousseaux, and G. Johansson, Transmon in a semi-infinite high-impedance transmission line: Appearance of cavity modes and Rabi oscillations, Phys. Rev. Res. 3, 023003 (2021).
[40] B. Richman and J. M. Taylor, Circulation by microwaveinduced vortex transport for signal isolation, PRX Quantum 2, 030309 (2021).

[41] M. Scheucher, A. Hilico, E. Will, J. Volz, and A Rauschenbeutel, Quantum optical circulator controlled by a single chirally coupled atom, Science 354, 1577 (2016).

[42] M. Jerger, Y. Reshitnyk, M. Oppliger, A. Potočnik, M. Mondal, A. Wallraff, K. Goodenough, S. Wehner, K. Juliusson, N. K. Langford, and A. Fedorov, Contextuality without nonlocality in a superconducting quantum system, Nat. Commun. 7, 12930 (2016).

[43] J. B. Johnson, Thermal agitation of electricity in conductors, Phys. Rev. 32, 97 (1928).

[44] H. Nyquist, Thermal agitation of electric charge in conductors, Phys. Rev. 32, 110 (1928).

[45] J. M. Fink, R. Bianchetti, M. Baur, M. Göppl, L. Steffen, S. Filipp, P. J. Leek, A. Blais, and A. Wallraff, Dressed Collective Qubit States and the Tavis-Cummings Model in Circuit QED, Phys. Rev. Lett. 103, 083601 (2009).

[46] A. Osman, J. Simon, A. Bengtsson, S. Kosen, P. Krantz, D. P. Lozano, M. Scigliuzzo, P. Delsing, J. Bylander, and A. Fadavi Roudsari, Simplified Josephson-junction fabrication process for reproducibly high-performance superconducting qubits, Appl. Phys. Lett. 118, 064002 (2021).

[47] C. D. Wilen, S. Abdullah, N. A. Kurinsky, C. Stanford, L. Cardani, G. D'Imperio, C. Tomei, L. Faoro, L. B. Ioffe, C. H. Liu, A. Opremcak, B. G. Christensen, J. L. DuBois, and R. McDermott, Correlated charge noise and relaxation errors in superconducting qubits, Nature (London) 594, 369 (2021).

[48] K. Serniak, M. Hays, G. de Lange, S. Diamond, S. Shankar, L. D. Burkhart, L. Frunzio, M. Houzet, and M. H. Devoret, Hot Nonequilibrium Quasiparticles in Transmon Qubits, Phys. Rev. Lett. 121, 157701 (2018).

[49] D. Ristè, C. C. Bultink, M. J. Tiggelman, R. N. Schouten, K. W. Lehnert, and L. DiCarlo, Millisecond charge-parity fluctuations and induced decoherence in a superconducting transmon qubit, Nat. Commun. 4, 1913 (2013).

[50] G. Catelani, J. Koch, L. Frunzio, R. J. Schoelkopf, M. H. Devoret, and L. I. Glazman, Quasiparticle Relaxation of Superconducting Qubits in the Presence of Flux, Phys. Rev. Lett. 106, 077002 (2011).

[51] G. Catelani, S. E. Nigg, S. M. Girvin, R. J. Schoelkopf, and L. I. Glazman, Decoherence of superconducting qubits caused by quasiparticle tunneling, Phys. Rev. B 86, 184514 (2012).

[52] K. Serniak, Nonequilibrium quasiparticles in superconducting qubits, Ph.D. thesis, Yale University, 2019.

[53] G. Catelani, R. J. Schoelkopf, M. H. Devoret, and L. I. Glazman, Relaxation and frequency shifts induced by quasiparticles in superconducting qubits, Phys. Rev. B 84, 064517 (2011).

[54] G. Catelani, Parity switching and decoherence by quasiparticles in single-junction transmons, Phys. Rev. B 89, 094522 (2014).

[55] J. M. Martinis, M. Ansmann, and J. Aumentado, Energy Decay in Superconducting Josephson-Junction Qubits from Nonequilibrium Quasiparticle Excitations, Phys. Rev. Lett. 103, 097002 (2009).

[56] U. Vool, I. M. Pop, K. Sliwa, B. Abdo, C. Wang, T. Brecht, Y. Y. Gao, S. Shankar, M. Hatridge, G. Catelani, M. Mirrahimi, L. Frunzio, R. J. Schoelkopf, L. I. Glazman, and M. H. Devoret, 
Non-Poissonian Quantum Jumps of a Fluxonium Qubit due to Quasiparticle Excitations, Phys. Rev. Lett. 113, 247001 (2014).

[57] C. Wang, Y. Y. Gao, I. M. Pop, U. Vool, C. Axline, T. Brecht, R. W. Heeres, L. Frunzio, M. H. Devoret, G. Catelani, L. I. Glazman, and R. J. Schoelkopf, Measurement and control of quasiparticle dynamics in a superconducting qubit, Nat. Commun. 5, 5836 (2014).

[58] E. T. Mannila, P. Samuelsson, S. Simbierowicz, J. T. Peltonen, V. Vesterinen, L. Grönberg, J. Hassel, V. F. Maisi, and J. P. Pekola, A superconductor free of quasiparticles for seconds, Nat. Phys. (2021), doi: 10.1038/s41567-021-01433-7.

[59] M. Houzet, K. Serniak, G. Catelani, M. H. Devoret, and L. I. Glazman, Photon-Assisted Charge-Parity Jumps in a Superconducting Qubit, Phys. Rev. Lett. 123, 107704 (2019).

[60] A. P. Vepsäläinen, A. H. Karamlou, J. L. Orrell, A. S. Dogra, B. Loer, F. Vasconcelos, D. K. Kim, A. J. Melville, B. M. Niedzielski, J. L. Yoder, S. Gustavsson, J. A. Formaggio, B. A. VanDevender, and W. D. Oliver, Impact of ionizing radiation on superconducting qubit coherence, Nature (London) 584, 551 (2020).

[61] L. Cardani, N. Casali, G. Catelani, T. Charpentier, M. Clemenza, I. Colantoni, A. Cruciani, L. Gironi, L. Gruenhaupt, D. Gusenkova, F. Henriques, M. Lagoin, M. Martinez, S. Pirro, I. M. Pop, C. Rusconi, A. Ustinov, F. Valenti, M. Vignati, and W. Wernsdorfer, DEMETRA: Suppression of the relaxation induced by radioactivity in superconducting qubits, J. Low Temp. Phys. 199, 475 (2020).

[62] I. V. Borzenets, U. C. Coskun, H. T. Mebrahtu, Y. V. Bomze, A. I. Smirnov, and G. Finkelstein, Phonon Bottleneck in Graphene-Based Josephson Junctions at Millikelvin Temperatures, Phys. Rev. Lett. 111, 027001 (2013).

[63] A. J. Ferguson, N. A. Court, F. E. Hudson, and R. G. Clark, Microsecond Resolution of Quasiparticle Tunneling in the
Single-Cooper-Pair Transistor, Phys. Rev. Lett. 97, 106603 (2006).

[64] M. Lenander, H. Wang, R. C. Bialczak, E. Lucero, M. Mariantoni, M. Neeley, A. D. O'Connell, D. Sank, M. Weides, J. Wenner, T. Yamamoto, Y. Yin, J. Zhao, A. N. Cleland, and J. M. Martinis, Measurement of energy decay in superconducting qubits from nonequilibrium quasiparticles, Phys. Rev. B 84, 024501 (2011).

[65] T. M. Stace, G. J. Milburn, and C. H. W. Barnes, Entangled two-photon source using biexciton emission of an asymmetric quantum dot in a cavity, Phys. Rev. B 67, 085317 (2003).

[66] C. Gardiner and P. Zoller, Quantum Noise: A Handbook of Markovian and non-Markovian Quantum Stochastic Methods with Applications to Quantum Optics (Springer, Berlin, 2004).

[67] C. K. Andersen and A. Blais, Ultrastrong coupling dynamics with a transmon qubit, New J. Phys. 19, 023022 (2017).

[68] A. Stockklauser, P. Scarlino, J. V. Koski, S. Gasparinetti, C. K. Andersen, C. Reichl, W. Wegscheider, T. Ihn, K. Ensslin, and A. Wallraff, Strong Coupling Cavity QED with Gate-Defined Double Quantum Dots Enabled by a High Impedance Resonator, Phys. Rev. X 7, 011030 (2017).

[69] M. T. Bell, J. Paramanandam, L. B. Ioffe, and M. E. Gershenson, Protected Josephson Rhombus Chains, Phys. Rev. Lett. 112, 167001 (2014).

[70] S. Fan, S. E. Kocabas, and J.-T. Shen, Input-output formalism for few-photon transport in one-dimensional nanophotonic waveguides coupled to a qubit, Phys. Rev. A 82, 063821 (2010).

[71] D. V. Nguyen, G. Catelani, and D. M. Basko, Dissipation in a superconducting artificial atom due to a single nonequilibrium quasiparticle, Phys. Rev. B 96, 214508 (2017).

[72] H. P. Breuer and F. Petruccione, The Theory of Open Quantum Systems (Oxford University Press, Oxford, 2002). 